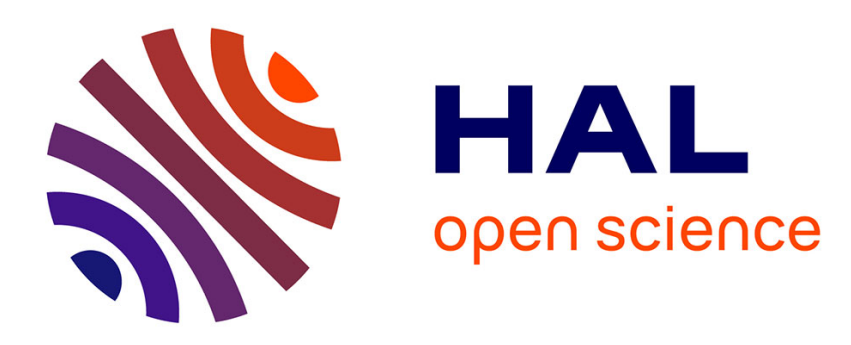

\title{
A Numerical Scheme for Impact Problems I: The One-Dimensional Case
}

Laetitia Paoli, Michelle Schatzman

\section{To cite this version:}

Laetitia Paoli, Michelle Schatzman. A Numerical Scheme for Impact Problems I: The One-Dimensional Case. SIAM Journal on Numerical Analysis, 2002, 40 (2), pp.702 - 733. 10.1137/S0036142900378728 . hal-01885729

\section{HAL Id: hal-01885729 \\ https://hal.science/hal-01885729}

Submitted on 2 Oct 2018

HAL is a multi-disciplinary open access archive for the deposit and dissemination of scientific research documents, whether they are published or not. The documents may come from teaching and research institutions in France or abroad, or from public or private research centers.
L'archive ouverte pluridisciplinaire HAL, est destinée au dépôt et à la diffusion de documents scientifiques de niveau recherche, publiés ou non, émanant des établissements d'enseignement et de recherche français ou étrangers, des laboratoires publics ou privés. 


\title{
A NUMERICAL SCHEME FOR IMPACT PROBLEMS I: THE ONE-DIMENSIONAL CASE*
}

\author{
LAETITIA PAOLI ${ }^{\dagger}$ AND MICHELLE SCHATZMAN ${ }^{\ddagger}$
}

\begin{abstract}
We consider a mechanical system with impact and one degree of freedom. The system is not necessarily Lagrangian. The representative point is subject to the constraint $u(t) \in \mathbb{R}^{+}$for all $t$. We assume that, at impact, the velocity is reversed and multiplied by a given coefficient of restitution $e \in[0,1]$. We define a numerical scheme which enables us to approximate the solutions of the Cauchy problem: this is an ad hoc scheme which does not require a systematic search for the times of impact. We prove the convergence of this numerical scheme to a solution. Many of the features of this proof will be reused in the nonconvex, multidimensional case, written in generalized coordinates, given in the companion paper [L. Paoli and M. Schatzman, SIAM J. Numer. Anal., 40 (2002), pp. 734-768]. We present some numerical results obtained with the scheme for a spring-dashpot system and we compare them to the results obtained by impact detection and penalization.
\end{abstract}

Key words. impact, coefficient of restitution, numerical scheme, convergence, local existence, global existence 47D03

AMS subject classifications. Primary, 65J10, 65M20, 65B05; Secondary, 17B09, 46N20,

PII. S0036142900378728

1. Introduction. We study in this article a numerical approximation of dynamics with impact with one degree of freedom when the representative point $u$ is subject to the constraint

$$
u \in K=[0, \infty) .
$$

Let $f$ be a continuous function from $[0, T] \times \mathbb{R} \times \mathbb{R}$ to $\mathbb{R}$ which is locally Lipschitz continuous with respect to its last two arguments.

The free dynamics of the system are written as

$$
\ddot{u}=f(\cdot, u, \dot{u}) .
$$

This system is more general than the system obtained in Lagrangian mechanics, since we want to include possible dissipative terms in the dynamics of the problem under discussion. There is no need for a mass matrix: in the one degree of freedom case, the velocity is always proportional to the impulsion, and an obvious change of variable enables us to forget about any other metric other than the Euclidean one.

Let us describe now the system satisfied by the problem with impact: we replace (1.1) with

$$
\ddot{u}=\mu+f(\cdot, u, \dot{u}),
$$

and since we cannot expect to have global solutions in general, $\mu$ is an unknown nonnegative measure on $\left[t_{0}, t_{0}+\bar{\tau}\right]$ which describes the reaction of the constraints; $\mu$

†UMR 5585 CNRS Analyse Numérique, Faculté des Sciences, Université Jean Monnet, 23 Rue du Docteur Paul Michelon, 42023 Saint-Etienne Cedex 2, France (paoli@anumsun1.univ-st-etienne.fr).

$\ddagger$ UMR 5585 CNRS Analyse Numérique, Université Lyon 1, 69622 Villeurbanne Cedex, France (schatz@maply.univ-lyon1.fr). 
has the following properties:

$$
\begin{aligned}
& \operatorname{supp}(\mu) \subset\left\{t \in\left[t_{0}, t_{0}+\bar{\tau}\right]: u(t)=0\right\}, \\
& \mu \geq 0 .
\end{aligned}
$$

We require the following functional properties for $u$ :

$$
\begin{aligned}
& u \text { is a continuous nonnegative function on }\left[t_{0}, t_{0}+\bar{\tau}\right], \\
& \dot{u} \text { is of bounded variation over }\left[t_{0}, t_{0}+\bar{\tau}\right] .
\end{aligned}
$$

We have to make a supplementary assumption in order to have a complete description of the impact; we choose a constitutive law of the impact using a coefficient of restitution. Thus we will assume that there exists $e \in[0,1]$ such that $\dot{u}(t+0)$ is equal to $-e$ times $\dot{u}(t-0)$. In other words, we have

$$
\dot{u}(t+0)=-e \dot{u}(t-0) .
$$

The set of admissible initial data $\mathbb{D}$ will be

$$
\mathbb{D}=\left\{\left(t_{0}, u_{0}, v_{0}\right) \in[0, T) \times K \times \mathbb{R}: \text { if } u_{0}=0, \text { then } v_{0} \geq 0\right\} .
$$

This choice is equivalent to the convention that there is no impact at the initial time $t_{0}$.

Given initial conditions $\left(t_{0}, u_{0}, v_{0}\right) \in \mathbb{D}$, we require that the following Cauchy data be satisfied:

$$
u\left(t_{0}\right)=u_{0}
$$

and

$$
\dot{u}\left(t_{0}\right)=v_{0} .
$$

For all initial data $\left(t_{0}, u_{0}, v_{0}\right) \in \mathbb{D}$ we will obtain the existence of a local solution to $(1.2),(1.3 \mathrm{a}),(1.3 \mathrm{~b})$, and (1.5) belonging to the functional class defined by (1.4a) and (1.4b) and satisfying the initial conditions (1.6) and (1.7).

The existence of this local solution is obtained by defining a numerical scheme, whose convergence will be shown in appropriate functional spaces; the limit of the approximation will be a solution of our problem. The projection on $K$ is given by

$$
P_{K}(x)=\max (x, 0)=x^{+} .
$$

Given two positive numbers $h^{*} \leq 1$ and $T$, assume that $F$ is a continuous function from $[0, T] \times \mathbb{R} \times \mathbb{R} \times \mathbb{R} \times\left[0, h^{*}\right]$ to $\mathbb{R}$, which is locally Lipschitz continuous with respect to its second, third, and fourth arguments. Assume, moreover, that $F$ is consistent with $f$, i.e., that for all $t \in[0, T]$, for all $u$ and $v$ in $\mathbb{R}$,

$$
F(t, u, u, v, 0)=f(t, u, v) .
$$

We approximate the solution of (1.2), (1.3a), (1.3b), and (1.4a), (1.4b), (1.5), (1.6), (1.7) by the following numerical scheme: the initial values $y^{0}$ and $y^{1}$ are given by the initial position

$$
y^{0}=u_{0},
$$


and the position at the first time step

$$
y^{1}=u_{0}+h v_{0}+h z(h),
$$

where $z(h)$ tends to 0 as $h$ tends to 0 .

Henceforth, we will systematically use the notation

$$
t^{m}=t_{0}+m h
$$

Given $y^{m-1}$ and $y^{m}, y^{m+1}$ is defined by the relations

$$
y^{m+1}=-e y^{m-1}+\left(2 y^{m}-(1-e) y^{m-1}+h^{2} F^{m}\right)^{+}
$$

and

$$
F^{m}=F\left(t^{m}, y^{m}, y^{m-1}, \frac{y^{m+1}-y^{m-1}}{2 h}, h\right)
$$

The reader should be aware at this point that, given $y^{m-1}$ and $y^{m}$, the existence of $y^{m+1}$ is an easy consequence of the strict contraction theorem; but we might be in trouble here, since $F$ is locally Lipschitz continuous and we are not sure about the existence of a solution on a finite time interval. This existence is not a trivial question and it depends on estimates which are at the heart of our subject.

A commentary on the construction of this scheme from the point of view of convex analysis will be useful here. We refer to the book of Rockafellar [25] for more information on the basic ideas in convex analysis to be used below.

Recall that the indicator function $\psi_{K}$ of the closed convex set $K$ is defined by

$$
\psi_{K}(x)= \begin{cases}0 & \text { if } x \in K \\ +\infty & \text { otherwise }\end{cases}
$$

and its subdifferential $\partial \psi_{K}$ is a function from $K$ to the set of closed convex sets given by

$$
\partial \psi_{K}(x)= \begin{cases}\{0\} & \text { if } x \in \operatorname{int}(K) \\ \mathbb{R}^{-} & \text {if } x \in \partial K\end{cases}
$$

For all $\lambda>0$, the multivalued equation

$$
x+\lambda \partial \psi_{K}(x) \ni f
$$

has a unique solution given by

$$
x=P_{K} f \text {. }
$$

In the announcement [22], we assumed that the set of constraints $K$ was convex and the geometry was Euclidean and $d$-dimensional, and we had defined a numerical scheme by the multivalued equation

$$
\frac{y^{m+1}-2 y^{m}+y^{m-1}}{h^{2}}+\partial \psi_{K}\left(\frac{y^{m+1}+e y^{m-1}}{1+e}\right) \ni F^{m} .
$$


We may rewrite (1.18) as

$$
\begin{aligned}
& \frac{y^{m+1}+e y^{m-1}}{1+e}+\frac{h^{2}}{1+e} \partial \psi_{K}\left(\frac{y^{m+1}+e y^{m-1}}{1+e}\right) \\
& \ni \frac{2 y^{m}-(1-e) y^{m-1}+h^{2} F^{m}}{1+e},
\end{aligned}
$$

which reduces, thanks to (1.16), (1.17), and (1.8), to relation (1.12). In contrast with the original proof of convergence of this scheme, as written in [19], the proof presented here is written in such a way that many of its features can be reused in the nonconvex, multidimensional case, written in generalized coordinates. This more general proof will be given in the companion paper [20].

Let us outline now the structure of the article and of the proofs. The main estimates are given by Lemma 2.1 in section 2 .

Then we find two constants $A$ and $\tau$ such that for initial data given by (1.10)(1.11), and for all small enough $h$ and all $m \leq \tau / h$, the discrete velocity is bounded:

$$
\sup \left|\left(y^{m+1}-y^{m}\right) / h\right| \leq A .
$$

In sections $4,5,6$, and 7 , we prove estimates on the discrete acceleration, we establish the variational properties of the limit of the numerical scheme, and we study the transmission of energy at impact, as well as the passage to the limit for the initial conditions. All these results are obtained under the assumption that on a certain time interval starting at $t_{0}$, the discrete velocity is bounded independently of the time step.

As a preliminary to the global existence proof, we give a priori estimates on problem (1.2)-(1.7) in section 8, which is completely independent from the remainder of the article.

In section 9 , we establish a very weak semicontinuity for the supremum of the local norm of the discrete velocities; this result enables us to obtain a global existence and convergence theorem.

This article contains theoretical results and reports also on some of the numerical implementations.

The existence result obtained here is a generalization of [26], [2], [24], [19], [17]. The numerical scheme has been implemented in the one-dimensional case, and the results were reported in [19], [18], [23]. In all these articles, we compared the performances of this scheme with those of a method based on the detection of impact. When the impact times are isolated, the algorithm by detection of impacts is more precise than the present scheme. As soon as the restitution coefficient is strictly less than one, we find systematically nonisolated impact times. In all cases, the present scheme is substantially faster. Since the phenomena that we want to approximate are highly nonlinear and often very sensitive to the initial data, the issue of precision is not necessarily crucial. Our numerical experiments show that the performance of the present numerical scheme is quite satisfactory from the point of view of qualitative conclusions.

The aforementioned references also concern the multidimensional case.

Let us remark that many articles have been devoted to the problem treated here under the assumption of inelasticity, i.e., a situation where the normal component of the impulsion vanishes after the impact. Moreau applied Gauss's principle of least constraint to unilateral problems in order to justify his choice of inelastic impact 
[11], which eventually led him to sweeping processes [14], followed by [12], [13]. Dry friction enters in Moreau's work as [15]; frictionless inelastic impact starts as [16], and the mathematical theory is tackled by M. Monteiro Marques in a series of articles: his main contributions are [9] for the general theory of differential inclusions, [10] for one-dimensional dynamics with friction, and [5] which adds percussion to the previous framework; this work is improved as [6], where dynamics of $n$ particles on a plane with normal friction are considered. The discretization approach has been taken up by Kunze and Monteiro Marques in [4], but most significantly by Stewart and Trinkle: they use that approach in [27], [29], and [30]. The real coronation is the beautiful and difficult article of Stewart [28], which concludes the study of dynamics with friction and inelastic impact for a finite number of degrees of freedom and one constraint; his results are not quite so precise in the case of many constraints but are still very important.

The philosophy of this long list of works is somewhat different from ours: we feel that not all impacts are inelastic, and we were originally motivated by continuous media; thus, we wanted to develop methods which work well for stiff systems of ordinary differential equations. From this point of view, any method which has to calculate with some precision the impact times is doomed to failure. On the other hand, the precision of the method presented here needs improvement, and globally it would make sense to agree on benchmarks which would enable the end-user to decide between different numerical methods.

Our approach is not the only possible one. In particular, in recent work, Mabrouk in [7] and [8] defines a numerical scheme for vibro-impact as a tool for proving existence of dynamics with impact; he allows for elastic, partially elastic, or inelastic impact. In his work, the mass matrix is assumed to be scalar, and therefore the metric is Euclidean. The idea is to discretize Moreau's formulation which describes the constraint in terms of velocity instead of describing it in terms of position, as we do here.

From a practical point of view a nice property of Mabrouk's scheme is that the velocity is reversed immediately upon impact. However, the number of steps during which the representative point of the system is outside of the set of constraints can be very large. For $e=0$, the representative point of the system can even leave for ever the set of constraints, while remaining close to it.

Therefore, numerical simulations will probably decide which of all these numerical schemes gives the most reliable simulations of dynamics with impact. It may well be that a future scheme will conciliate the two distinct approaches and perform better than them: the road is open to researchers to try their ingenuity on these challenging problems.

2. The heart of the estimates. In the one-dimensional case, the main estimate on the numerical scheme is described in the following lemma; we recall the definition

$$
r^{+}=\max (r, 0) .
$$

LEMma 2.1. Let the real-valued sequence $\left(y^{m}\right)_{m}$ satisfy the following recurrence relation for all $m \geq 1$ :

$$
y^{m+1}=-e y^{m-1}+\left(2 y^{m}-(1-e) y^{m-1}\right)^{+}+h^{2} \lambda^{m} .
$$

Then, for all $m \geq 2$, the discrete velocity

$$
\eta^{m}=\left(y^{m+1}-y^{m}\right) / h
$$


satisfies the estimate

$$
\left|\eta^{m}\right| \leq \max \left(\left|\eta^{m-1}\right|, e\left|\eta^{m-2}\right|\right)+h\left|\lambda^{m}\right|+h\left|\lambda^{m-1}\right| .
$$

Proof. Assume first that $2 y^{m}-(1-e) y^{m-1}$ is nonnegative, and substitute $y^{m+1}=$ $y^{m}+h \eta^{m}, y^{m-1}=y^{m}-h \eta^{m-1}$ into (2.1); we obtain

$$
\eta^{m}=\eta^{m-1}+h \lambda^{m}
$$

so that

$$
\left|\eta^{m}\right| \leq\left|\eta^{m-1}\right|+h\left|\lambda^{m}\right|
$$

Assume now that $2 y^{m}-(1-e) y^{m-1}$ is strictly negative. On the one hand, (2.1) implies the relation

$$
\eta^{m}=e \eta^{m-1}-\frac{1+e}{h} y^{m}+h \lambda^{m}
$$

the assumption on the sign of $2 y^{m}-(1-e) y^{m-1}$ is equivalent to

$$
\frac{(1+e) y^{m}}{h}<-(1-e) \eta^{m-1}
$$

and therefore

$$
\eta^{m}>\eta^{m-1}+h \lambda^{m}
$$

On the other hand, we subtract from the relation

$$
y^{m+1}+e y^{m-1}=h^{2} \lambda^{m}
$$

the inequality implied by (2.1) with $m$ substituted by $m-1$ :

$$
y^{m}+e y^{m-2} \geq h^{2} \lambda^{m-1},
$$

and we infer that

$$
\eta^{m} \leq-e \eta^{m-2}+h\left(\lambda^{m}-\lambda^{m-1}\right) .
$$

When we summarize (2.4), (2.5), and (2.6), we find (2.3).

\section{Existence of a discrete solution and estimates on the discrete veloc-} ity. We systematically use the floor and ceiling notations: when $r$ is a real number, the floor $\lfloor r\rfloor$ of $r$ is the largest integer at most equal to $r$, and the ceiling $\lceil r\rceil$ is the smallest integer at least equal to $r$. In this section we prove that for $h$ and $\tau$ small enough, relations (1.12) and (1.13) uniquely define a numerical solution while $(m+1) h \leq \tau$; moreover the discrete velocity of this solution is bounded independently of $h$.

The idea of this result is to show the existence by the Brouwer fixed point argument and the uniqueness by local considerations.

We say that a pair of numbers $y^{0}$ and $y^{1}$ satisfy the property $P(a, h)$ if the following conditions are true:

$$
\begin{aligned}
& \left|y^{0}\right| \leq a, \quad\left|y^{1}\right| \leq a \\
& \left|y^{1}-y^{0}\right| \leq a h, \\
& \left\{\begin{array}{c}
\text { There exists } y^{2} \text { such that }\left|y^{2}-y^{1}\right| \leq a h \\
\text { and } y^{2}+e y^{0}-\left(2 y^{1}-(1-e) y^{0}+h^{2} F\left(t^{1}, y^{0}, y^{1},\left(y^{2}-y^{0}\right) / 2 h, h\right)\right)^{+}=0 .
\end{array}\right.
\end{aligned}
$$


Theorem 3.1. For all $a>0$ and for all $A>a$, there exists $\tau>0$ such that, for all $t_{0} \in[0, T)$ and for all $y^{0}$ and $y^{1}$ satisfying property $P(a, h)$, there exists a numerical solution of the scheme (1.12) and (1.13) for $0 \leq m h \leq \tau$, which satisfies, moreover, the estimate

$$
\forall m \in\{0, \ldots,\lfloor\tau / h\rfloor-1\}, \quad\left|y^{m+1}-y^{m}\right| \leq A h .
$$

Proof. Define

$$
C_{1}=\sup \left\{\left|F\left(t, u, u^{\prime}, 0, h\right)\right|: 0 \leq t \leq T, \quad|u| \leq a, \quad\left|u^{\prime}\right| \leq a, \quad 0 \leq h \leq h^{*}\right\} .
$$

Let $L$ be the local Lipschitz constant of $F$ defined by

$$
\begin{aligned}
& \forall\left(t, u_{i}, u_{i}^{\prime}, v_{i}, h\right) \in[0, T] \times[-a-A T, a+A T]^{2} \times[-A, A] \times\left[0, h^{*}\right], \quad i=1,2, \\
& \left|F\left(t, u_{1}, u_{1}^{\prime}, v_{1}, h\right)-F\left(t, u_{2}, u_{2}^{\prime}, v_{2}, h\right)\right| \leq L\left(\left|u_{1}-u_{2}\right|+\left|u_{1}^{\prime}-u_{2}^{\prime}\right|+\left|v_{1}-v_{2}\right|\right) .
\end{aligned}
$$

Choose $\tau>0$ such that

$$
2 \tau\left(C_{1}+L A+2 L A \tau\right) \leq A-a .
$$

We will apply a Brouwer fixed point argument; we choose $y^{2}$ according to (3.1c), and we define a compact convex set $B_{h}$ by

$$
\begin{aligned}
B_{h}=\left\{\widehat{y}=\left(\widehat{y}^{m}\right)_{0 \leq m h \leq \tau}: \widehat{y}^{0}=y^{0}, \widehat{y}^{1}=y^{1}, \widehat{y}^{2}=y^{2},\right. \\
\\
\left.\forall m \in\{1, \ldots,\lfloor\tau / h\rfloor-1\}:\left|\widehat{y}^{m+1}-\widehat{y}^{m}\right| \leq A h\right\} .
\end{aligned}
$$

Assuming that $\widehat{y}$ belongs to $B_{h}$, we define $\widehat{F}$ by

$$
\widehat{F}^{m}=F\left(t^{m}, \widehat{y}^{m}, \widehat{y}^{m-1},\left(\widehat{y}^{m+1}-\widehat{y}^{m-1}\right) / 2 h, h\right), \quad m \in\{1, \ldots,\lfloor\tau / h\rfloor-1\} .
$$

We now write the numerical scheme

$$
y^{m+1}+e y^{m-1}-\left(2 y^{m}-(1-e) y^{m-1}+h^{2} \widehat{F}^{m}\right)^{+}=0,
$$

which can be put under the form (2.1), provided that we define

$$
h^{2} \lambda^{m}=\left(2 y^{m}-(1-e) y^{m-1}+h^{2} \widehat{F}^{m}\right)^{+}-\left(2 y^{m}-(1-e) y^{m-1}\right)^{+} .
$$

It should be remarked that (3.4) possesses a unique solution, since it is explicit in $y^{m+1}$, and that if the mapping $\widehat{y} \mapsto y$ possesses a fixed point, this fixed point is precisely the numerical solution sought here. We estimate the discrete velocity $\eta^{m}$ thanks to Lemma 2.1: the number $\lambda^{m}$ is estimated by

$$
\begin{aligned}
& \left|\lambda^{m}\right| \leq\left|\widehat{F}^{m}\right| \\
& \leq\left|F\left(t^{m}, y^{0}, y^{0}, 0, h\right)\right|+L\left(\left|\widehat{y}^{m}-y^{0}\right|+\left|\widehat{y}^{m-1}-y^{0}\right|+\left|\widehat{\eta}^{m}\right| / 2+\left|\widehat{\eta}^{m-1}\right| / 2\right),
\end{aligned}
$$

and the assumption $\widehat{y} \in B_{h}$ guarantees that

$$
\left|\lambda^{m}\right| \leq C_{1}+2 L A \tau+L A .
$$

Estimate (2.3) implies

$$
\left|\eta^{m}\right| \leq \max \left(\left|\eta^{0}\right|,\left|\eta^{1}\right|\right)+2\left(C_{1}+2 L A \tau+L A\right) m h
$$


by discrete integration. We may conclude now that for $(m+1) h \leq \tau$,

$$
\left|\eta^{m}\right| \leq a+2\left(C_{1}+2 L A \tau+L A\right) \tau,
$$

and thanks to assumption (3.3), $y$ also belongs to $B_{h}$. This mapping is clearly continuous, which implies the existence of a fixed point thanks to Brouwer's fixed point theorem.

There remains two easy lemmas; the first one settles for $h$ small the question of the uniqueness of the numerical solution.

Lemma 3.2. Under the hypotheses of Theorem 3.1, there exists $h_{1}>0$ such that for all $h \in\left(0, h_{1}\right]$, for all $y^{0}$ and $y^{1}$ satisfying condition $P(a, h)$, the numerical solution of (1.12) and (1.13) satisfying estimate (3.2) is unique.

Proof. Given $y^{m-1}$ and $y^{m}$, the discrete velocity $\eta^{m}$ is a fixed point of the mapping

$$
\begin{aligned}
\eta & \mapsto h^{-1}\left(-e y^{m-1}-y^{m}\right. \\
& \left.+\left(2 y^{m}-(1-e) y^{m-1}+h^{2} F\left(t^{m}, y^{m}, y^{m-1},\left(\eta+\eta^{m-1}\right) / 2, h\right)\right)^{+}\right) .
\end{aligned}
$$

Let $L$ be the Lipschitz constant of the mapping

$$
z \mapsto F\left(t, y, y^{\prime}, z, h\right)
$$

for $t \in[0, T], y$ and $y^{\prime}$ in $[-a-T A, a+T A],|z| \leq A$, and $0 \leq h \leq h^{*}$. Then the Lipschitz constant of the mapping (3.7) is $h L / 2$, and therefore, if $h_{1}<2 / M$, the uniqueness of $\eta^{m}$ is guaranteed and the lemma is proved.

The second lemma establishes that under conditions (1.10) and (1.11), property $P(a, h)$ holds.

Lemma 3.3. Assume that $y^{0}=u_{0}$ and $y^{1}=u_{0}+h v_{0}+h o(h)$ as in (1.11); then there exists $h_{1}>0$ such that for all $h \in\left(0, h_{1}\right]$, there exists a unique $y^{2}$ such that

$$
y^{2}+e y^{0}-\left(2 y^{1}-(1-e) y^{0}+h^{2} F\left(t^{1}, y^{0}, y^{1},\left(y^{2}-y^{0}\right) / 2 h, h\right)\right)^{+}=0
$$

and

$$
\max \left(\left|y^{1}-y^{0}\right|,\left|y^{2}-y^{1}\right|\right) \leq\left(3\left|v_{0}\right|+1\right) h .
$$

Proof. Define a mapping

$$
z \mapsto h^{-1}\left(\left((1+e) y^{0}+2 \eta^{0} h+h^{2} F\left(t^{1}, y^{0}, y^{1}, z / 2, h\right)\right)^{+}-(1+e) y^{0}\right),
$$

which is slightly different from the mapping (3.7), since its fixed point will be $\left(y^{2}-\right.$ $\left.y^{0}\right) / h$. Standard arguments show that it is possible to find $h_{1}>0$ such that for all $h \in\left(0, h_{1}\right]$ and all $y^{1}$ in $\left[y^{0}-1, y^{0}+1\right]$, the mapping (3.8) is a strict contraction from the ball of radius $2\left|v_{0}\right|+1 / 2$ to itself. We set $y^{2}=y^{0}+h z$, where $z$ is the fixed point of the above mapping. Then it is clear that for $h$ small enough,

$$
\left|\eta^{2}\right|=\left|\left(y^{2}-y^{1}\right) / h\right| \leq|z|+\left|\eta^{0}\right|,
$$

and the lemma is proved.

As a consequence of Theorem 3.1 and Lemma 3.2, we have the following result. 
Proposition 3.4. For all $\left(t_{0}, u_{0}, v_{0}\right) \in \mathbb{D}$, there exists $A>0, \tau \in\left(0, T-t_{0}\right]$, and $h_{1} \in\left(0, h^{*}\right]$ such that for all $m \in\{0, \ldots,\lfloor\tau / h\rfloor\}, y^{m}$ is uniquely defined by (1.10), (1.11), and the recursive formulas (1.12), (1.13) and satisfies the estimate

$$
\forall m \in\{0, \ldots,\lfloor\tau / h\rfloor-1\}, \quad\left|y^{m+1}-y^{m}\right| \leq A h .
$$

Proof. The main observation is that we have to choose $A$ as a function of the initial data. Thanks to Lemma 3.3, it suffices to take

$$
A \geq \max \left(3\left|v_{0}\right|+1,\left|u_{0}\right|+1\right)
$$

the remainder of the argument is clear.

4. Estimates on the discrete acceleration. In this section and the three following ones, we assume that there exist strictly positive numbers $\tau, A$, and $h_{1}$ and a subsequence of time steps to which correspond solutions of the numerical scheme defined by (1.10), (1.11), (1.12), and (1.13), which satisfy the estimate, for all $h \leq h_{1}$,

$$
\forall l \in\{0, \ldots, P-1\}, \quad\left|y^{l+1}-y^{l}\right| \leq A h,
$$

where

$$
P=\lfloor\tau / h\rfloor .
$$

Here we estimate the discrete total variation of the sequence $\left(\eta^{m}\right)_{m}$.

THEOREM 4.1. Under assumption (4.1), there exists a constant $C_{2}$ such that for all $h \leq h_{1}$

$$
\sum_{m=1}^{P-1}\left|\eta^{m}-\eta^{m-1}\right| \leq C_{2} .
$$

Proof. The constant $C_{3}$ is taken as a majorant of $\left|F^{m}\right|$; we can take it as equal to

$$
\begin{gathered}
C_{3}=\max \left\{\left|F\left(t, y, y^{\prime}, z, h\right)\right|: t \in[0, T],\left|y-u_{0}\right| \leq A T,\right. \\
\left.\left|y^{\prime}-u_{0}\right| \leq A T,|z| \leq A, 0 \leq h \leq h^{*}\right\} .
\end{gathered}
$$

We put the numerical scheme under the form (2.1) by defining $\lambda^{m}$ through

$$
h^{2} \lambda^{m}=\left(2 y^{m}-(1-e) y^{m-1}+h^{2} F^{m}\right)^{+}-\left(2 y^{m}-(1-e) y^{m-1}\right)^{+},
$$

which differs slightly from (3.5), since it involves $F^{m}$ instead of $\widehat{F}^{m}$. The number $\lambda^{m}$ is estimated by

$$
\left|\lambda^{m}\right| \leq\left|F^{m}\right| \leq C_{3}
$$

We observe that

$$
\eta^{m}-\eta^{m-1}=h \lambda^{m}+\left(2 y^{m}-(1-e) y^{m-1}\right)^{-} / h .
$$

Therefore, by the triangle inequality,

$$
\left|\eta^{m}-\eta^{m-1}\right| \leq h C_{3}+\left(2 y^{m}-(1-e) y^{m-1}\right)^{-} / h .
$$


Using (4.5) again, we obtain

$$
\left|\eta^{m}-\eta^{m-1}\right| \leq 2 h C_{3}+\eta^{m}-\eta^{m-1} .
$$

We observe that we have the elements of a telescoping sum: we sum (4.6) for $m$ varying from 1 to $P-1$ and we get

$$
\sum_{m=1}^{P-1}\left|\eta^{m}-\eta^{m-1}\right| \leq 2 h C_{3} P+\eta^{P-1}-\eta^{0} \leq 2 C_{3} \tau+2 A .
$$

5. Variational properties of the limit of the numerical scheme. In this section, we work under the assumption (4.1). We define a function $u_{h}$ by affine interpolation, as follows:

$$
\left\{\begin{aligned}
& u_{h}(t)= y^{m}+\left(t-t_{0}-m h\right) \frac{y^{m+1}-y^{m}}{h} \\
& \text { for } t-t_{0} \in[m h,(m+1) h), 0 \leq m \leq P-1, \\
& u_{h}(t)=y^{P} \quad \text { for } t-t_{0} \in[P h, \tau] .
\end{aligned}\right.
$$

We also define a measure $F_{h}$ as the following sum of Dirac masses:

$$
F_{h}(t)=\sum_{m=1}^{P-1} h F^{m} \delta\left(t-t_{0}-m h\right) .
$$

In this section we prove that the sequence $\left(u_{h}\right)_{h}$ converges in an appropriate sense to a function $u$ which satisfies (1.2) to (1.4b) with $\tau$ instead of $\bar{\tau}$. We delay the proof of (1.5), the transmission condition at impacts, to a later section.

There are three steps in the convergence proof: the first is to prove that the limit $u$ exists in an appropriate sense and takes its values in $K$; in the second step, we show that $\dot{u}_{h}$ is of bounded variation uniformly in $h$ and that $F_{h}$ converges to $f(\cdot, u, \dot{u})$ weakly in the space of $\mathbb{R}$-valued measures. The last step is the characterization of the measure $\mu=\ddot{u}-f(\cdot, u, \dot{u})$ : there we show that $\mu$ satisfies conditions (1.3a) and (1.3b).

Lemma 5.1. From all sequence of functions $\left(u_{h}\right)_{h}$ indexed by a sequence $h$ tending to 0 , it is possible to extract a subsequence, still denoted by $\left(u_{h}\right)_{h}$, such that

$$
\begin{aligned}
& u_{h} \rightarrow u \quad \text { in } C^{0}\left(\left[t_{0}, t_{0}+\tau\right]\right) \text { strong }, \\
& \dot{u}_{h} \rightarrow \dot{u} \quad \text { in } L^{\infty}\left(\left[t_{0}, t_{0}+\tau\right]\right) \text { weak }{ }^{*} .
\end{aligned}
$$

The function u takes its values in $K$.

Proof. Thanks to assumption (4.1), we know that $\left(u_{h}\right)_{0<h \leq h_{1}}$ is uniformly Lipschitz continuous over $\left[t_{0}, t_{0}+\tau\right]$. Therefore, we may extract a subsequence, still denoted by $u_{h}$, such that (5.3) and (5.4) hold. Thus $u$ belongs to $W^{1, \infty}\left(\left[t_{0}, t_{0}+\tau\right]\right) \cap$ $C^{0}\left(\left[t_{0}, t_{0}+\tau\right]\right)$, which means that $u$ is a Lipschitz continuous function [1]. For all $m$ belonging to $\{1, \ldots, P-1\}$, we have that

$$
\frac{y^{m+1}+e y^{m-1}}{1+e}=y^{m}+h \frac{\eta^{m}-e \eta^{m-1}}{1+e} \geq 0 .
$$

It follows that, for all $m \in\{1, \ldots, P-1\}$, the Euclidean distance between $y^{m}$ and $K$ can be estimated as follows:

$$
\left(y^{m}\right)^{-} \leq h\left|\eta^{m}-e \eta^{m-1}\right| /(1+e) \leq h A .
$$


Thanks to the definition (5.1), we can see that for all $t \in\left[t_{0}, t_{0}+\tau\right]$ the Euclidean distance between $u_{h}(t)$ and $K$ is estimated by $2 h A$. This allows us to pass to the limit when $h$ tends to 0 and to conclude.

The next lemma describes the convergence of the measures involved in our problem; we denote by $M^{1}\left(\left(t_{0}, t_{0}+\tau\right)\right)$ the space of bounded measures over $\left(t_{0}, t_{0}+\tau\right)$.

Lemma 5.2. The measures $\ddot{u}_{h}$ and $F_{h}$ converge weakly $*$ in $M^{1}\left(\left(t_{0}, t_{0}+\tau\right)\right)$ to $\ddot{u}$ and $f(\cdot, u, \dot{u})$, respectively.

Proof. The measure $\ddot{u}_{h}$ is a sum of Dirac measures on $\left(t_{0}, t_{0}+\tau\right)$; more precisely, we have

$$
\ddot{u}_{h}(t)=\sum_{m=1}^{P-1}\left(\eta^{m}-\eta^{m-1}\right) \delta\left(t-t_{0}-m h\right)-\eta^{P-1} \delta\left(t-t_{0}-P h\right),
$$

and the total variation of $\dot{u}_{h}$ on $\left(t_{0}, t_{0}+\tau\right)$ is estimated by

$$
T V\left(\dot{u}_{h}\right) \leq \sum_{m=1}^{P-1}\left|\eta^{m}-\eta^{m-1}\right|+\left|\eta^{P-1}\right| .
$$

Theorem 4.1 implies that $\left(\dot{u}_{h}\right)_{0<h \leq h_{1}}$ is a bounded family in $B V\left(\left(t_{0}, t_{0}+\tau\right)\right)$, the space of functions of bounded variation over $\left(t_{0}, t_{0}+\tau\right)$. Using Helly's theorem, we can extract another subsequence $\left(\dot{u}_{h}\right)_{h}$ which converges, except perhaps on a countable set of points, to a function of bounded variation. Hence

$$
\dot{u} \in B V\left(\left(t_{0}, t_{0}+\tau\right)\right) .
$$

Moreover,

$$
\ddot{u}_{h} \rightarrow \ddot{u} \quad \text { weakly } * \text { in } M^{1}\left(\left(t_{0}, t_{0}+\tau\right)\right) .
$$

Lebesgue's theorem implies that $\dot{u}_{h}$ converges to $\dot{u}$ in $L^{1}\left(t_{0}, t_{0}+\tau\right)$. We extend $\dot{u}_{h}$ and $\dot{u}$ to $\mathbb{R}$ by 0 outside of $\left(t_{0}, t_{0}+\tau\right)$ and still denote the respective extensions by $\dot{u}_{h}$ and $\dot{u}$. The set $\left\{\dot{u}_{h}: h \in\left(0, h_{1}\right]\right\} \cup\{\dot{u}\}$ is a compact subset of $L^{1}(\mathbb{R})$. The classical characterization of compact subsets of $L^{1}(\mathbb{R})[3]$ implies that

$$
\lim _{\theta \rightarrow 0} \sup _{0<h \leq h_{1}} \int_{\mathbb{R}}\left|\dot{u}_{h}(t-\theta)-\dot{u}_{h}(t)\right| d t=0 .
$$

Letting $\theta=h$, we can see that $\dot{u}_{h}(\cdot-h)$ converges to $\dot{u}$ in $L^{1}(\mathbb{R})$. Let us define an approximate velocity $v_{h}$ on $\mathbb{R}$ by

$$
v_{h}(t)=\frac{\dot{u}_{h}(t-h+0)+\dot{u}_{h}(t+0)}{2} .
$$

The sequence $v_{h}$ converges to $\dot{u}$ in $L^{1}(\mathbb{R})$. Moreover, for all $t \in\left[t^{m}, t^{m+1}\right)$ and for all $m \in\{1, \ldots, P-1\}$, we have the identity

$$
v_{h}(t)=\frac{\eta^{m}+\eta^{m-1}}{2} .
$$

We immediately have the following estimates for all $t \in\left(t_{0}, t_{0}+\tau\right)$ and all $h \in\left(0, h_{1}\right]$ :

$$
\left|v_{h}(t)\right| \leq A, \quad\left|u_{h}(t)-u_{0}\right| \leq A\left(t-t_{0}\right) \leq A \tau .
$$


Let $\psi$ be a continuous function over $[0, T]$ with compact support included in $\left(t_{0}, t_{0}+\tau\right)$. For all small enough $h$, the support of $\psi$ is included in $\left[t_{0}+h, t_{0}+(P-1) h\right]$. The duality product $\left\langle F_{h}, \psi\right\rangle$ has the expression

$$
\left\langle F_{h}, \psi\right\rangle=\sum_{m=1}^{P-1} h \psi\left(t_{0}+m h\right) F^{m} .
$$

We wish to compare the expression (5.8) to

$$
\int_{t_{0}}^{t_{0}+\tau} \psi f(\cdot, u, \dot{u}) d t
$$

We compare the right-hand side of (5.8) which is basically a numerical quadrature by the formula of rectangles to an appropriate integral. Let us rewrite the individual terms of the right-hand side of (5.8) as

$$
h \psi\left(t^{m}\right) F^{m}=\int_{t^{m}}^{t^{m+1}} \psi(t) F^{m} d t+\int_{t^{m}}^{t^{m+1}}\left(\psi\left(t^{m}\right)-\psi(t)\right) F^{m} d t .
$$

Consider now the second term on the right-hand side of (5.10). Recalling estimate (4.3),

$$
\max _{0 \leq m \leq n}\left|F^{m}\right| \leq C_{3},
$$

and denoting by $\omega_{\psi}$ the modulus of continuity of $\psi$ we can see that

$$
\left|\int_{t^{m}}^{t^{m+1}}\left(\psi\left(t^{m}\right)-\psi(t)\right) F^{m} d t\right| \leq C_{3} \omega_{\psi}(h) h .
$$

We consider now the first term on the right-hand side of (5.10), which we would like to compare to expression (5.9). Thanks to the consistency assumption (1.9) we have the following inequalities, for all $t \in\left[t^{m}, t^{m+1}\right)$ and all $m \in\{1, \ldots, P-1\}$ :

$$
\begin{aligned}
\mid F^{m} & -f\left(t, u_{h}(t), v_{h}(t)\right) \mid \\
& \leq\left|F\left(t^{m}, y^{m}, y^{m-1}, v_{h}\left(t^{m}\right), h\right)-F\left(t^{m}, u_{h}(t), u_{h}(t), v_{h}\left(t^{m}\right), h\right)\right| \\
& +\left|F\left(t^{m}, u_{h}(t), u_{h}(t), v_{h}\left(t^{m}\right), h\right)-F\left(t^{m}, u_{h}(t), u_{h}(t), v_{h}(t), 0\right)\right| \\
& +\left|f\left(t^{m}, u_{h}(t), v_{h}(t)\right)-f\left(t, u_{h}(t), v_{h}(t)\right)\right| .
\end{aligned}
$$

Denote by $\mathcal{D}$ the set

$$
\begin{gathered}
\mathcal{D}=\left\{\left(t, u_{1}, u_{2}, v, h\right): 0 \leq t \leq T, \quad\left|u_{1}-u_{0}\right| \leq A T,\right. \\
\left.\left|u_{2}-u_{0}\right| \leq A T, \quad|v| \leq A, \quad 0 \leq h \leq h^{*}\right\} .
\end{gathered}
$$

Let $L$ be the Lipschitz constant of $\left(u_{1}, u_{2}\right) \mapsto F\left(t, u_{1}, u_{2}, v, h\right)$ restricted to $\mathcal{D}$ and let $\omega_{F}$ be the modulus of continuity of $F$ on $\mathcal{D}$. With these notations, we can see that

$$
\begin{aligned}
& \left|F^{m}-f\left(t, u_{h}(t), v_{h}(t)\right)\right| \\
& \quad \leq L\left(\left|y^{m}-u_{h}(t)\right|+\left|y^{m-1}-u_{h}(t)\right|\right)+2 \omega_{F}(h) .
\end{aligned}
$$


Since $\left(u_{h}\right)_{h}$ converges strongly in $C^{0}\left(\left[t_{0}, t_{0}+\tau\right]\right)$ and $\left(v_{h}\right)_{h}$ converges strongly to $\dot{u}$ in $L^{1}(\mathbb{R})$ and almost everywhere on $\left(t_{0}, t_{0}+\tau\right)$, we see that $f\left(\cdot, u_{h}, v_{h}\right)$ tends to $f(\cdot, u, \dot{u})$ strongly in $L^{1}\left(t_{0}, t_{0}+\tau\right)$ and almost everywhere on $\left(t_{0}, t_{0}+\tau\right)$. We summarize relations (5.12) and (5.13) together with the above convergence result, and we find that

$$
\begin{aligned}
& \left|\left\langle F_{h}, \psi\right\rangle-\int_{t_{0}}^{t_{0}+\tau} \psi f(\cdot, u, \dot{u}) d t\right| \\
& \leq \int_{t_{0}}^{t_{0}+\tau}\left|f\left(\cdot, u_{h}, v_{h}\right)-f(\cdot, u, \dot{u})\right||\psi| d t \\
& \quad+C_{3} \omega_{\psi}(h) \tau+\left(3 L A h+2 \omega_{F}(h)\right) \int_{t_{0}}^{t_{0}+\tau}|\psi| d t
\end{aligned}
$$

which concludes the proof.

Let us prove now that the measure $\mu$ has the required variational properties.

LEMma 5.3. The measure $\mu$ satisfies properties (1.3a) and (1.3b).

Proof. Define

$$
\mu_{h}=\ddot{u}_{h}-F_{h}
$$

$\mu_{h}$ is a sum of Dirac measures on $\left(t_{0}, t_{0}+\tau\right)$. More precisely

$$
\begin{aligned}
\mu_{h}= & \sum_{m=1}^{P-1}\left(\eta^{m}-\eta^{m-1}-h F^{m}\right) \delta\left(t-t_{0}-m h\right) \\
& -\eta^{P-1} \delta\left(t-t_{0}-P h\right) .
\end{aligned}
$$

With all the previous results, we know that $\mu_{h}$ converges to $\mu=\ddot{u}-f(\cdot, u, p)$ weakly * in $M^{1}\left(\left(t_{0}, t_{0}+\tau\right)\right)$. Let us prove property (1.3a). Assume that $\tau_{0}$ is a point of $\left(t_{0}, t_{0}+\tau\right)$ such that $u\left(\tau_{0}\right)>0$. Then, by continuity of $u$, there exist $\varepsilon>0$ and $\rho>0$ such that

$$
\forall t \in\left(\tau_{0}-\varepsilon, \tau_{0}+\varepsilon\right), \quad u(t) \geq 3 \rho .
$$

Since the sequence $\left(u_{h}\right)_{h}$ converges uniformly to $u$ as $h$ tends to 0 , we can decrease $h_{1}$ so that

$$
\forall h \in\left(0, h_{1}\right], \quad \forall t \in\left(\tau_{0}-\varepsilon, \tau_{0}+\varepsilon\right), \quad u_{h}(t) \geq 2 \rho .
$$

Replacing $y^{m-1}$ by $y^{m}-h \eta^{m-1}$, we have

$$
2 y^{m}-(1-e) y^{m-1}+h^{2} F^{m}=(1+e) y^{m}+(1-e) h \eta^{m-1}+h^{2} F^{m},
$$

and relations (4.3) and (4.1) imply that

$$
2 y^{m}-(1-e) y^{m-1}+h^{2} F^{m} \geq(1+e) y^{m}-(1-e) h A-h^{2} C_{3} .
$$

Possibly decreasing $h_{1}$, we have

$$
\forall h \in\left(0, h_{1}\right], \quad \forall t^{m} \in\left(\tau_{0}-\varepsilon, \tau_{0}+\varepsilon\right), \quad 2 y^{m}-(1-e) y^{m-1}+h^{2} F^{m} \geq \rho,
$$

and thus

$$
\forall h \in\left(0, h_{1}\right], \quad \forall t^{m} \in\left(\tau_{0}-\varepsilon, \tau_{0}+\varepsilon\right), \quad \eta^{m}-\eta^{m-1}-h F^{m}=0 .
$$


This proves that, for $h$ small enough, the support of $\mu_{h}$ does not intersect the open set $\left(\tau_{0}-\varepsilon, \tau_{0}+\varepsilon\right)$ and therefore relation (1.3a). In order to conclude the proof, we observe that

$$
\eta^{m}-\eta^{m-1}-h F^{m}=\frac{1}{h}\left(2 y^{m}-(1-e) y^{m-1}+h^{2} F^{m}\right)^{-} \geq 0 .
$$

Thus, for all $\tau^{\prime} \in(0, \tau)$, the measure $\mu_{h}$ is nonnegative on $\left(t_{0}, t_{0}+\tau^{\prime}\right)$ for $h$ small enough, which implies by a straightforward passage to the limit that $\mu$ is nonnegative. This concludes the proof of the lemma.

6. Transmission of energy during impact. The basic assumption is still the one made at the beginning of section 4 .

Let $\bar{\tau} \in(0, \tau)$ be such that $u\left(t_{0}+\bar{\tau}\right)$ vanishes. Write $\bar{t}=t_{0}+\bar{\tau}$.

We will prove the relation

$$
\dot{u}(\bar{t}+0)=-e \dot{u}(\bar{t}-0)
$$

by performing a precise analysis of the transmission of the energy by the scheme.

Possibly decreasing $h_{1}$, there exists a nonempty interval $\left[\tau_{-5}, \tau_{2}\right]$ containing $\bar{\tau}$ and included in $[h,(P-1) h]$. The apparently strange notations $\tau_{-5}$ and $\tau_{2}$ have been chosen in view of the upcoming construction of Lemmas 6.1 and 6.2, where we will consider relative times

$$
\tau_{-5}<\cdots<\tau_{-1}<\bar{\tau}<\tau_{1}<\tau_{2}
$$

Define

$$
P=\left\lceil\tau_{-5} / h\right\rceil+1 \text { and } Q=\left\lfloor\tau_{2} / h\right\rfloor-1 .
$$

The measure $\ddot{u}_{h}$ is a sum of Dirac measures on $\left(t_{0}+\tau_{-5}, t_{0}+\tau_{2}\right)$. We define two measures $\omega_{h}$ and $\lambda_{h}$ on $\left(t_{0}+\tau_{-5}, t_{0}+\tau_{2}\right)$ by

$$
\omega_{h}(t)=\sum_{m=P}^{Q} \frac{\left(-2 y^{m}+(1-e) y^{m-1}\right)^{+}}{h} \delta\left(t-t_{0}-m h\right)
$$

and

$$
\lambda_{h}(t)=\sum_{m=P}^{Q} h \lambda^{m} \delta\left(t-t_{0}-m h\right) .
$$

We have

$$
\ddot{u}_{h}=\omega_{h}+\lambda_{h},
$$

and it is obvious that $\omega_{h}$ is a nonnegative measure.

Since the real numbers $\lambda^{m}$ are bounded independently of $h$ and $m$, the measure by $\left|\lambda_{h}\right|$ of any subinterval $[a, b]$ of $\left(t_{0}+\tau_{-5}, t_{0}+\tau_{2}\right)$ is bounded by $C_{3}(b-a+h)$, and it is clear therefore that there exists a function $\lambda \in L^{\infty}\left(t_{0}+\tau_{-5}, t_{0}+\tau_{2}\right)$ and a subsequence $\lambda_{h}$ converging to $\lambda$ in the weak * topology of $M^{1}\left(\left(t_{0}+\tau_{-5}, t_{0}+\tau_{2}\right)\right)$.

The measure $\omega_{h}$ converges in the weak * topology of $M^{1}\left(\left(t_{0}+\tau_{-5}, t_{0}+\tau_{2}\right)\right)$ to a nonnegative measure $\omega$ and in the limit

$$
\ddot{u}=\omega+\lambda,
$$


while

$$
|\lambda|_{L^{\infty}} \leq C_{3} .
$$

Since $u$ is nonnegative on $\left(t_{0}+\tau_{-5}, t_{0}+\tau_{2}\right)$ and $u(\bar{t})$ vanishes, we must have

$$
\dot{u}(\bar{t}+0) \geq 0, \quad \dot{u}(\bar{t}-0) \leq 0 .
$$

On the other hand, $\dot{u}(\bar{t}+0)-\dot{u}(\bar{t}-0)$ is equal to $\omega(\{\bar{t}\})$; if $\omega(\{\bar{t}\})$ vanishes, we have

$$
\dot{u}(\bar{t}+0)=\dot{u}(\bar{t}-0)=0,
$$

and the identity

$$
\dot{u}(\bar{t}+0)=-e \dot{u}(\bar{t}-0)
$$

holds. Therefore, the only interesting case is when

$$
\omega(\{\bar{t}\})>0 .
$$

The following two lemmas enable us to prove in two steps that the velocity is reversed according to the law described by (1.5). Lemma 6.1 shows that if $\omega$ has a Dirac mass at $\bar{t}$, then the left velocity at $\bar{t}$ is outgoing; Lemma 6.2 indeed shows that (1.5) holds.

LEMma 6.1. If $\omega(\{\bar{t}\})$ is strictly positive, then $\dot{u}(\bar{t}-0)$ is strictly negative.

Proof. The idea of the proof is to find two successive times $t^{m-1} \leq t^{m}<\bar{t}$ for which we can write down an estimate on the discrete velocities and then to use Lemma 2.1 to perform a discrete integration and to obtain a contradiction. We must deal with the fact that $\dot{u}_{h}$ does not converge uniformly to $\dot{u}$.

Without loss of generality, we may assume that $\dot{u}$ is continuous on the right and that for all $h \leq h_{1}, \dot{u}_{h}$ is also continuous from the right. According to Helly's theorem, there exists a countable set $D$ such that

$$
\dot{u}_{h}(t) \rightarrow \dot{u}(t) \quad \forall t \text { such that } t-\bar{t} \in\left(\tau_{-5}, \tau_{2}\right) \backslash D .
$$

Assume that $\dot{u}(\bar{t}-0)$ vanishes; therefore, $\dot{u}(\bar{t}+0)$ is strictly positive. Choose $\alpha=$ $\dot{u}(\bar{t}+0) / 4$, and let $\tau_{-4}$ and $\tau_{1}$ be such that

$$
\begin{aligned}
& \tau_{-5} \leq \tau_{-4}<\bar{\tau}<\tau_{1} \leq \tau_{2}, \\
& 6 C_{3}\left(\tau_{1}-\tau_{-4}\right) \leq \alpha,
\end{aligned}
$$

and

$$
\omega\left(\left[t_{0}+\tau_{-4}, \bar{t}\right)\right) \leq \alpha, \quad \omega\left(\left(\bar{t}, t_{0}+\tau_{1}\right]\right) \leq \alpha .
$$

An integration of (6.1) on appropriate intervals yields

$$
\begin{aligned}
\forall t \in\left(t_{0}+\tau_{-4}, \bar{t}\right), & |\dot{u}(t \pm 0)| \leq \alpha+C_{3}(\bar{t}-t), \\
\forall t \in\left(\bar{t}, t_{0}+\tau_{1}\right), \quad & \dot{u}(t \pm 0) \geq 2 \omega(\{\bar{t}\})-\alpha-C_{3}(t-\bar{t}) .
\end{aligned}
$$

Choose $\tau_{-3} \in\left(\tau_{-4}, \bar{\tau}\right) \backslash D$ and $\tau_{-1} \in\left(\tau_{-3}, \bar{\tau}\right) \backslash D$; since $\omega_{h}$ is a nonnegative measure, we have the following inequality for all $\tau^{\prime} \in\left(\tau_{-3}, \tau_{-1}\right)$ and all $\tau^{\prime \prime} \in\left(\tau^{\prime}, \tau_{-1}\right)$ :

$$
\begin{aligned}
& \left|\dot{u}_{h}\left(t_{0}+\tau^{\prime}\right)-\dot{u}_{h}\left(t_{0}+\tau^{\prime \prime}\right)\right| \leq \omega_{h}\left(\left(t_{0}+\tau^{\prime}, t_{0}+\tau^{\prime \prime}\right]\right)+C_{3}\left(\tau^{\prime \prime}-\tau^{\prime}+h\right) \\
& \quad \leq \omega_{h}\left(\left[t_{0}+\tau_{-3}, t_{0}+\tau_{-1}\right]\right)+C_{3}\left(\tau^{\prime \prime}-\tau^{\prime}+h\right) .
\end{aligned}
$$


We integrate $\omega_{h}-\omega$ on the interval $\left[t_{0}+\tau_{-3}, t_{0}+\tau_{-1}\right]$; since the measures $\omega$ and $\omega_{h}$ do not charge $t_{0}+\tau_{-3}$ and $t_{0}+\tau_{-1}$, we find that

$$
\begin{aligned}
& \omega_{h}\left(\left[t_{0}+\tau_{-3}, t_{0}+\tau_{-1}\right]\right)-\omega\left(\left[t_{0}+\tau_{-3}, t_{0}+\tau_{-1}\right]\right) \\
& \quad=\dot{u}_{h}\left(t_{0}+\tau_{-1}\right)-\dot{u}_{h}\left(t_{0}+\tau_{-3}\right)-\dot{u}\left(t_{0}+\tau_{-1}\right)+\dot{u}\left(t_{0}+\tau_{-3}\right) \\
& \quad+\lambda\left(\left[t_{0}+\tau_{-3}, t_{0}+\tau_{-1}\right]\right)-\lambda_{h}\left(\left[t_{0}+\tau_{-3}, t_{0}+\tau_{-1}\right]\right)
\end{aligned}
$$

and therefore

$$
\begin{aligned}
& \omega_{h}\left(\left[t_{0}+\tau_{-3}, t_{0}+\tau_{-1}\right]\right) \\
& \quad \leq \omega\left(\left[t_{0}+\tau_{-3}, t_{0}+\tau_{-1}\right]\right)+\left|\dot{u}_{h}\left(t_{0}+\tau_{-1}\right)-\dot{u}\left(t_{0}+\tau_{-1}\right)\right| \\
& \quad+\left|\dot{u}_{h}\left(t_{0}+\tau_{-3}\right)-\dot{u}\left(t_{0}+\tau_{-3}\right)\right|+C_{3}\left(2\left(\tau_{-1}-\tau_{-3}\right)+h\right) .
\end{aligned}
$$

Choose now $\tau_{-2} \in\left(\tau_{-3}, \tau_{-1}\right) \backslash D$; then, for $h$ small enough, $t^{m}=h\left\lfloor\tau_{2} / h\right\rfloor$ and $t^{m-1}=t^{m}-h$ belong to the interval $\left(\tau_{-3}, \tau_{-1}\right)$, and therefore

$$
\left|\dot{u}_{h}\left(t^{m}\right)-\dot{u}_{h}\left(t^{m-1}\right)\right| \leq \alpha+C_{3}\left(2\left(\tau_{-1}-\tau_{-3}\right)+3 h\right)+\varepsilon_{h}
$$

where $\varepsilon_{h}$ tends to 0 as $h$ tends to 0 . On the other hand, $\dot{u}_{h}\left(t_{0}+\tau_{-2}\right)$ tends to $\dot{u}\left(t_{0}+\tau_{-2}\right)$ and therefore, thanks to relation (6.6), there exists a family $\varepsilon_{h}^{\prime}$ such that

$$
\left|\dot{u}_{h}\left(t_{0}+\tau_{-2}\right)\right|=\left|\dot{u}_{h}\left(t^{m}\right)\right| \leq \alpha+C_{3}\left(\bar{\tau}-\tau_{-2}\right)+\varepsilon_{h}^{\prime},
$$

which is equivalent to

$$
\left|\eta^{m}\right| \leq \alpha+C_{3}\left(\bar{\tau}-\tau_{-2}\right)+\varepsilon_{h}^{\prime}
$$

we infer from (6.8) and (6.9) that

$$
\left|\eta^{m-1}\right| \leq 2 \alpha+C_{3}\left(2\left(\tau_{-1}-\tau_{-3}\right)+\bar{\tau}-\tau_{-2}+3 h\right)+\varepsilon_{h}+\varepsilon_{h}^{\prime} .
$$

Thus, for all $n \geq m$ we infer from Lemma 2.1 that

$$
\begin{aligned}
\left|\eta^{n}\right| \leq & 2 \alpha+C_{3}\left(2\left(\tau_{-1}-\tau_{-3}\right)+3 h\right. \\
& \left.+\bar{\tau}-\tau_{-2}+2\left(t^{n}-t^{m}\right)\right)+\varepsilon_{h}+\varepsilon_{h}^{\prime} .
\end{aligned}
$$

Therefore, in the limit, for all $t \geq t_{0}+\tau_{-2}$

$$
|\dot{u}(t)| \leq 2 \alpha+C_{3}\left(2\left(\tau_{-1}-\tau_{-3}\right)+\bar{\tau}-\tau_{-2}+2\left(t-t_{0}-\tau_{-2}\right)\right),
$$

and for all $t \in\left[t_{0}+\tau_{-2}, t_{0}+\tau_{1}\right]$

$$
|\dot{u}(t)| \leq 2 \alpha+C_{3}\left(2\left(\tau_{-1}-\tau_{-3}\right)+\bar{\tau}-\tau_{-2}+2\left(\tau_{1}-\tau_{-2}\right)\right) .
$$

On the other hand, relation (6.7) implies that for all $t \in\left(\bar{t}, t_{0}+\tau_{1}\right)$,

$$
|\dot{u}(t)| \geq 3 \alpha-C_{3}\left(\tau_{1}-\bar{\tau}\right) .
$$

Under assumption (6.4), relation (6.11) contradicts relation (6.10).

We can conclude now the local study of the reflection of the velocity by the following lemma. 
Lemma 6.2. If $\omega(\{\bar{t}\})$ is strictly positive, then

$$
\dot{u}(\bar{t})=-e \dot{u}(\bar{t}-0) .
$$

Proof. Since $\dot{u}(\bar{t}-0)$ is strictly negative, there exists a real number $\tau_{-3}$ such that $u(t)$ is strictly positive on $\left[t_{0}+\tau_{-3}, \bar{t}\right) \subset\left[t_{0}+\tau_{-5}, \bar{t}\right)$. For all $\tau_{-2} \in\left(\tau_{-3}, \bar{\tau}\right)$, there exists $\tau_{-1} \in\left(\tau_{-2}, \bar{\tau}\right)$ and $h_{1}>0$ such that

$$
\forall h \in\left(0, h_{1}\right], \quad \forall t \in\left[t_{0}+\tau_{-2}, t_{0}+\tau_{-1}\right), \quad u_{h}(t) \geq \frac{u\left(t_{0}+\tau_{-2}\right)}{2} .
$$

We prove now that there exists a maximal integer

$$
m \in\left\{\left\lfloor\tau_{-3} / h\right\rfloor, \ldots,\left\lfloor\left(\tau_{2}\right) / h\right\rfloor\right\}
$$

such that

$$
\forall l \in\left\{\left\lfloor\tau_{-3} / h\right\rfloor, \ldots, m-1\right\}, \quad 2 y^{l}-(1-e) y^{l-1} \geq 0,
$$

and denoting

$$
\rho_{h}=t^{m-1}-t_{0}
$$

the time $\rho_{h}$ satisfies

$$
\lim _{h \rightarrow 0} \rho_{h}=\bar{\tau} .
$$

Let us first observe that for all small enough $h$ and all $t_{l}$ belonging to $\left[t_{0}+\tau_{-2}, t_{0}+\tau_{-1}\right)$ we have

$$
2 y^{l}-(1-e) y^{l-1} \geq 0
$$

Indeed,

$$
\begin{aligned}
2 y^{l}-(1-e) y^{l-1} & =(1+e) y^{l}+(1-e) h \eta^{l-1} \\
& \geq \frac{1+e}{2} u\left(t_{0}+\tau_{-2}\right)-h(1-e) A,
\end{aligned}
$$

and if $2 A(1-e) h \leq(1+e) u\left(t_{0}+\tau_{-2}\right)$, we can see that (6.17) holds. Therefore $m$ exists and

$$
\liminf \rho_{h} \geq \bar{\tau} .
$$

On the other hand, if there existed $\tau_{1}>\bar{\tau}$ such that for all $t^{m} \in\left[t_{0}+\tau_{-3}, t_{0}+\right.$ $\tau_{1}$ ] we had (6.17), then $\omega_{h}$ would vanish on $\left(t_{0}+\tau_{-3}, t_{0}+\tau_{1}\right)$, which contradicts assumption (6.3). Therefore, we have shown that

$$
\lim \sup \rho_{h} \leq \bar{\tau},
$$

i.e., (6.16). We integrate (4.4) discretely, and we find that for $t \in\left[t_{0}+\tau_{-3}, t_{0}+\rho_{h}\right]$

$$
\begin{aligned}
u_{h}(t)= & u_{h}\left(t_{0}+\rho_{h}\right)-\left(t_{0}+\rho_{h}-t\right) \dot{u}_{h}\left(t_{0}+\rho_{h}\right) \\
& +\int_{t}^{t_{0}+\rho_{h}} \lambda_{h}\left(\left(s, t_{0}+\rho_{h}\right]\right) d s .
\end{aligned}
$$


In the limit we have

$$
u(t)=u\left(t_{0}+\bar{\tau}\right)-\left(\bar{\tau}+t_{0}-t\right) \lim _{h \downarrow 0} \dot{u}_{h}\left(t_{0}+\rho_{h}+0\right)+\int_{t}^{t_{0}+\bar{\tau}} \int_{s}^{t_{0}+\bar{\tau}} \lambda(r) d r d s .
$$

The comparison of (6.18) and (6.19) shows that

$$
\lim _{h \downarrow 0} \dot{u}_{h}\left(t_{0}+\rho_{h}+0\right)=\lim _{h \downarrow 0} \eta^{m-1}=\dot{u}(\bar{t}-0) .
$$

Our purpose now is to obtain very precise estimates on the behavior of $y_{h}$ beyond $t_{0}+\rho_{h}$. Thanks to the maximality of $m$, we have the relation

$$
y^{m+1}=-e y^{m-1}+h^{2} \lambda^{m}
$$

let us estimate $2 y^{m+1}-(1-e) y^{m}$. We substitute the value of $y^{m+1}$ given by $(6.21)$ into this expression, and we also use (4.4) with $m$ replaced by $m-1$; we find that

$$
\begin{aligned}
& 2 y^{m+1}-(1-e) y^{m} \\
& \quad=-\left[2 y^{m-1}-(1-e) y^{m-2}\right]-(1-e) h^{2} \lambda^{m-1}+2 h^{2} \lambda^{m} .
\end{aligned}
$$

We apply relation (2.1) for $n=m+1$ and we find that

$$
\begin{aligned}
& \eta^{m+1}+e \eta^{m-1}=h\left(\lambda^{m+1}-\lambda^{m}\right) \\
& \quad+\left\{-\left[2 y^{m-1}-(1-e) y^{m-2}\right] h^{-1}-(1-e) h \lambda^{m-1}+2 h \lambda^{m}\right\}^{+} .
\end{aligned}
$$

Therefore, we have

$$
\eta^{m+1}+e \eta^{m-1} \geq-2 h C_{3} .
$$

On the other hand, if $\xi=-\left[2 y^{m-1}-(1-e) y^{m-2}\right] h^{-1}-(1-e) h \lambda^{m-1}+2 h \lambda^{m}$ is less than or equal to 0 ,

$$
\left|\eta^{m+1}+e \eta^{m-1}\right| \leq 2 h C_{3}
$$

if $\xi$ is positive, then the sign condition on $2 y^{m-1}-(1-e) y^{m-2}$ implies that

$$
\eta^{m+1}+e \eta^{m-1} \leq h\left(\lambda^{m+1}+\lambda^{m}\right)-(1-e) h \lambda^{m-1} .
$$

Thus, we have shown that

$$
\left|\eta^{m+1}+e \eta^{m-1}\right| \leq 3 C_{3} h .
$$

If $e$ is strictly positive, then for all small enough $h$,

$$
\eta^{m+1} \geq e|\dot{u}(\bar{t}-0)| / 2 .
$$

Let us estimate now the expression $2 y^{m+2}-(1-e) y^{m+1}$ : we have

$$
2 y^{m+2}-(1-e) y^{m+1}=-e\left[2 y^{m}-(1-e) y^{m-1}\right]+O\left(h^{2}\right) .
$$

If $2 y^{m+2}-(1-e) y^{m+1}$ is nonnegative, then

$$
y^{m+3}=2 y^{m+2}-y^{m+1}+h^{2} \lambda^{m+2} .
$$


We must estimate $2 y^{m+3}-(1-e) y^{m+2}$ :

$$
\begin{aligned}
& 2 y^{m+3}-(1-e) y^{m+2}-2 y^{m+2}+(1-e) y^{m+1} \\
& =h\left(2 \eta^{m+2}-(1-e) \eta^{m+1}\right) \\
& =h(1+e) \eta^{m+1}+2 h^{2} \lambda^{m+2}
\end{aligned}
$$

and therefore $2 y^{m+3}-(1-e) y^{m+2}$ is nonnegative for all small enough $h$; the repetition of the argument shows that there exists $\theta>0$ such that for all small enough $h$ and all $n \in\{m+2, \ldots, m+\lfloor\theta / h\rfloor\}$, the expression $2 y^{n+1}-(1-e) y^{n}$ is nonnegative, and thus we have the relations

$$
y^{n}=y^{m+1}+h(n-m-1) \eta^{m+1}+\sum_{j=m+2}^{n-1}(n-j) h^{2} \lambda^{j} .
$$

On the other hand, if $2 y^{m+2}-(1-e) y^{m+1}$ is negative, we must have

$$
y^{m}=-\frac{(1-e) h \eta^{m-1}}{1+e}+O\left(h^{2}\right)
$$

and therefore

$$
y^{m-1}=-\frac{2 h \eta^{m-1}}{1+e}+O\left(h^{2}\right)
$$

These relations and the assumption on the sign of $2 y^{m+2}-(1-e) y^{m+1}$ imply that

$$
2 y^{m+3}-(1-e) y^{m+2}=-\frac{\left(4 e^{2}+e(1-e)^{2}\right) h \eta^{m-1}}{1+e}+O\left(h^{2}\right),
$$

which is strictly positive for $h$ small enough. But now, we can see that

$$
y^{m+3}-y^{m+2}=-e h \eta^{m-1}+O\left(h^{2}\right),
$$

which is strictly positive for small enough $h$, and therefore $2 y^{m+4}-(1-e) y^{m+3}$ is strictly positive for $h$ small enough, since

$$
2 y^{m+4}-(1-e) y^{m+3} \geq-h e(1+e) \eta^{m-1}+O\left(h^{2}\right)
$$

the same argument as above shows now that there exists $\theta>0$ such that for all $n \in\{m+3, \ldots, m+\lfloor\theta / h\rfloor\}$,

$$
y^{n}=y^{m+2}+h(n-m-2) \eta^{m+2}+\sum_{j=m+3}^{n-1}(n-j) h^{2} \lambda^{j} .
$$

If we let $\rho_{h}^{\prime}=t^{m+1}-t_{0}$ in the first case and $\rho_{h}^{\prime}=t^{m+2}-t_{0}$ in the second case, we have now for $\rho_{h}^{\prime} \leq t-t_{0} \leq \rho_{h}^{\prime}+\theta-h$

$$
u_{h}(t)=u_{h}\left(t_{0}+\rho_{h}^{\prime}\right)+\left(t-\rho_{h}^{\prime}-t_{0}\right) \dot{u}_{h}\left(t_{0}+\rho_{h}^{\prime}\right)+\int_{t_{0}+\rho_{h}^{\prime}}^{t} \lambda_{h}((s, t]) d s
$$

and

$$
u_{h}\left(t_{0}+\rho_{h}^{\prime}\right)=O(h), \quad \dot{u}_{h}\left(t_{0}+\rho_{h}^{\prime}\right)=-e \eta^{m-1}+O(h) .
$$


Passing to the limit in (6.24), we can see that

$$
\dot{u}(\bar{t}+0)=-e \dot{u}(\bar{t}-0)
$$

If we assume now that $e$ vanishes, relation (6.22) implies

$$
\eta^{m+1}=O(h)
$$

We observe that Lemma 2.1 implies that for all $n$

$$
\left|\eta^{n}\right| \leq\left|\eta^{n-1}\right|+2 C_{3} h
$$

which implies immediately that for $n \geq m+1$

$$
\left|\eta^{n}\right| \leq\left|\eta^{m+1}\right|+2 h C_{3}(n-m-1),
$$

which proves by a straightforward passage to the limit that

$$
\dot{u}(\bar{t}+0)=0 .
$$

This completes the proof of the lemma.

7. Initial conditions. In this section we prove that the solution that we have constructed satisfies the initial conditions; we work under the hypotheses stated at the beginning of section 4 .

LEMma 7.1. The function $u$ satisfies the initial conditions

$$
u\left(t_{0}\right)=u_{0}, \quad \dot{u}\left(t_{0}+0\right)=v_{0} .
$$

Proof. By uniform convergence of $u_{h}$ to $u$, it is clear that $u\left(t_{0}\right)$ is equal to $u_{0}$. There remains to show that the initial condition on the velocity is satisfied.

Assume first $u_{0}>0$; then there exist $h_{1}>0$ and $\tau_{1}>0$ such that for all $h \in\left(0, h_{1}\right]$ and for all $t-t_{0} \in\left[0, \tau_{1}\right]$

$$
u_{h}(t) \geq u_{0} / 2
$$

Then, for all $t^{m}-t_{0} \in\left(0, \tau_{1}\right], 2 y^{m}-(1-e) y^{m-1}+h^{2} F^{m}$ belongs to $K$ for $h$ small enough; we indeed have

$$
\begin{aligned}
2 y^{m}-(1-e) y^{m-1}+h^{2} F^{m} & \geq(1+e) y^{m}-(1-e) h A-h^{2} C_{3} \\
& \geq(1+e) u_{0} / 2-(1-e) h A-h^{2} C_{3}
\end{aligned}
$$

which is strictly positive for $h$ small enough. Thus the constraints are not active for $t_{0} \leq t^{m} \leq t_{0}+\tau_{1}$ and the convergence is clear.

In the second case, $u_{0}$ vanishes; we have taken admissible initial conditions, so that

$$
v_{0} \geq 0
$$

Let us show that

$$
\dot{u}\left(t_{0}+0\right)=v_{0},
$$


considering two cases: $v_{0}>0$ and $v_{0}=0$. When $v_{0}$ vanishes, we have

$$
y^{1}=y^{0}+h v_{0}+O\left(h^{2}\right)=O\left(h^{2}\right)
$$

and

$$
\begin{aligned}
y^{2} & =-e y^{0}+\left(2 y^{1}-(1-e) y^{0}\right)^{+}+h^{2} \lambda^{1} \\
& =2\left(y^{1}\right)^{+}+h^{2} \lambda^{1}=O\left(h^{2}\right) .
\end{aligned}
$$

Thus,

$$
\eta^{0}=O(h), \quad \eta^{1}=O(h),
$$

and relation (2.3) implies

$$
\left|\eta^{m}\right| \leq O(h)+2 C_{3} h(m-1)
$$

therefore, a passage to the limit immediately gives

$$
\dot{u}\left(t_{0}+0\right)=0 .
$$

If, on the other hand, $v_{0}$ is strictly positive, then

$$
2 y^{1}-(1-e) y^{0}=2 y^{1}=2 h v_{0}+O\left(h^{2}\right),
$$

which is strictly positive if $h$ is small enough. Let $\{1, \ldots, m\}$ be the maximal interval such that

$$
2 y^{n}-(1-e) y^{n-1}>0 \quad \text { if } n \leq m .
$$

Then, for all $n \in\{1, \ldots, m\}$,

$$
\eta^{n}-\eta^{n-1}=h \lambda^{n},
$$

which implies by discrete integration that

$$
\eta^{n} \geq \eta^{0}-h n C_{3},
$$

as long as $n$ belongs to $\{1, \ldots, m\}$. Moreover, if we choose any $\tau_{1}<v_{0} /\left(2 C_{3}\right)$ and if $n$ is at most equal to $\min \left(m,\left\lfloor\tau_{1} / h\right\rfloor\right)$, we can see that

$$
y^{n}=y_{0}+h\left(\eta^{0}+\cdots+\eta^{n-1}\right) \geq \frac{h n v_{0}}{2}
$$

for all small enough values of $h$.

In particular, for all $n \leq \min \left(m,\left\lfloor\tau_{1} / h\right\rfloor\right)$,

$$
2 y^{n}-(1-e) y^{n-1} \geq \frac{(1+e) h n v_{0}}{2}-(1-e) h A,
$$

which proves that $m$ is at least equal to $\left\lfloor\tau_{1} / h\right\rfloor$. Therefore, $\omega_{h}$ vanishes on the interval $\left(t_{0}, t_{0}+\tau_{1}-h\right)$; in the limit, $\omega$ vanishes on $\left(t_{0}, t_{0}+\tau_{1}\right)$ and therefore

$$
\dot{u}\left(t_{0}+0\right)=v_{0},
$$

which completes the proof of the lemma. 
8. A priori estimates. In this section we prove that solutions of the problem (1.2), (1.3a), (1.3b), (1.4a), (1.4b), (1.5), (1.6), and (1.7) satisfy an a priori estimate on an interval with nonempty interior.

Lemma 8.1. Let $R$ be strictly larger than $\left|v_{0}\right|$. Then there exists $\tau(R)>0$ such that for all solution $u$ of (1.2), (1.3a), (1.3b), (1.4a), (1.4b), (1.5), (1.6), and (1.7) defined on $\left[t_{0}, t_{0}+\tau\right]$, the following estimates hold:

$$
\forall t \in\left[t_{0}, t_{0}+\min (\tau, \tau(R))\right], \quad\left|u(t)-u_{0}\right| \leq R, \quad|\dot{u}(t)| \leq R .
$$

Proof. The measure $\mu$ appearing in (1.3b) can be decomposed in the sum of an atomic part $\mu_{a}$ and a diffuse part $\mu_{d}$. There might be a continuous singular part in the measure $\mu$, and it is convenient to see $\mu$ as the sum of the derivative of a jump function and of the derivative of a continuous function. At each point of the support of $\mu_{a}$ we have

$$
|\dot{u}(t+0)| \leq|\dot{u}(t-0)|
$$

thanks to relation (1.5). On any interval $\left(t_{1}, t_{2}\right)$ which does not intersect the support of $\mu_{a}$, we multiply relation (1.2) by $\dot{u}$, and we find that

$$
\frac{d}{d t} \frac{1}{2}|\dot{u}|^{2}=\dot{u} f(\cdot, u, \dot{u}) .
$$

Define

$$
z=|\dot{u}| \text {. }
$$

Relations (8.2) and (8.3) imply that in the sense of measures

$$
z \dot{z} \leq \dot{u} f(\cdot, u, \dot{u}) .
$$

Our purpose now is to transform (8.4) into a differential inequality. We write

$$
\dot{u} f(t, u, \dot{u})=\dot{u}\left[f(t, u, \dot{u})-f\left(t, u_{0}, 0\right)+f\left(t, u_{0}, 0\right)\right] .
$$

Define

$$
g(t)=\left|f\left(t, u_{0}, 0\right)\right|,
$$

fix $R>\left|v_{0}\right|$, and let $\omega(R)$ be the Lipschitz constant of $(u, v) \mapsto f(t, u, v)$ for $t \in[0, T]$ and $\max \left(\left|u-u_{0}\right|,|v|\right) \leq R$. By construction, $\omega$ is continuous and it is an increasing function of $R$.

If $t_{0} \leq t \leq t_{0}+\tau$ and if $\max \left(\left|u(t)-u_{0}\right|,|\dot{u}(t)|\right) \leq R$ on $\left[t_{0}, t_{0}+\tau\right]$, we have the inequality

$$
z \dot{z} \leq|\dot{u} f(\cdot, u, \dot{u})| \leq z\left(g+\omega(R)\left(\left|u-u_{0}\right|+|\dot{u}|\right)\right) .
$$

But we can estimate $u(t)-u_{0}$ :

$$
\left|u(t)-u_{0}\right| \leq \int_{t_{0}}^{t}|\dot{u}(s)| d s \leq \int_{t_{0}}^{t} z d s .
$$

Therefore we have the estimate

$$
|\dot{u} f(\cdot, u, \dot{u})| \leq z g+z \omega(R)\left(\int_{t_{0}}^{t} z d s+z\right),
$$


and we conclude that $z$ satisfies the differential inequality

$$
\dot{z} \leq g+\omega(R)\left[\int_{t_{0}}^{t} z d s+z\right]
$$

Let $\widehat{z}$ be the solution of the integro-differential equation

$$
\frac{d \widehat{z}}{d t}=g+\omega(R)\left[\int_{t_{0}}^{t} \widehat{z} d s+\widehat{z}\right], \quad \widehat{z}\left(t_{0}\right)=\left|v_{0}\right| .
$$

Such a $\widehat{z}$ exists and is unique, by very classical arguments, and it is also a majorant of $z$. Let $\tau(R)$ be the largest number in $\left(0, T-t_{0}\right]$ such that

$$
\forall t \in\left[t_{0}, t_{0}+\tau(R)\right], \quad \widehat{z}(t) \leq R, \quad \int_{t_{0}}^{t} \widehat{z}(s) d s \leq R .
$$

Such a number exists since $\widehat{z}\left(t_{0}\right)$ is strictly inferior to $R$. On the interval $\left[t_{0}\right.$, $\left.t_{0}+\min (\tau, \tau(R))\right]$ we have the desired estimate.

9. Global results. We summarize the results obtained so far in the following proposition.

Proposition 9.1. Assume that there exist strictly positive numbers $\tau, A$, and $h_{1}>0$ and a sequence of solutions of the numerical scheme defined by (1.10), (1.11), (1.12), and (1.13), which satisfies the estimate (4.1). Then it is possible to extract from the sequence $u_{h}$ defined by (5.1) a subsequence which converges to a solution of (1.2), (1.3a), (1.3b), (1.4a), (1.4b), (1.5), (1.6), and (1.7). The convergence holds in the following sense: $u_{h}$ converges uniformly to $u_{h}$ on $\left[t_{0}, t_{0}+\tau\right] ; \dot{u}_{h}$ converges to $\dot{u}$ in $L^{\infty}\left(t_{0}, t_{0}+\tau\right)$ weakly $*$ and almost everywhere on $\left[t_{0}, t_{0}+\tau\right]$; and $\ddot{u}_{h}$ converges to $\ddot{u}$ in the weak $*$ topology of measures. Moreover, for all $\bar{\tau} \in(0, \tau]$, we have the following convergence:

$$
\begin{aligned}
& \limsup _{h \downarrow 0} \sup \left\{\left|\eta^{m}\right|: t_{0} \leq t^{m} \leq t_{0}+\bar{\tau}\right\} \\
& \quad \leq \operatorname{ess} \sup \left\{|\dot{u}(t)|: t_{0} \leq t \leq t_{0}+\bar{\tau}\right\} .
\end{aligned}
$$

Proof. The only statement which deserves a proof is the last one; if it is not true, there exists $\gamma>0$, a sequence of time steps still denoted by $h$, and a sequence of integers $m(h)$ such that

$$
\left|\eta^{m(h)}\right| \geq \operatorname{ess} \sup \left\{|\dot{u}(t)|: t_{0} \leq t \leq t_{0}+\bar{\tau}\right\}+\gamma .
$$

Without loss of generality, we may assume that $h m(h)$ tends to $\tau_{2} \in[0, \bar{\tau}]$.

First, $\tau_{2}$ cannot be equal to 0 : we have learnt in section 7 that there exists a constant $C_{4}$ and a time $\tau_{1}$ such that for all $h \leq h_{1}$ and all $m \leq \tau_{1} / h$,

$$
\left|\eta^{m}-\eta^{0}\right| \leq C_{4} m h
$$

In particular, this estimate implies that

$$
\left|\eta^{m(h)}\right|=\left|v_{0}\right|+O(m h)
$$

but $\left|v_{0}\right|$ is at most equal to ess $\sup \left\{|\dot{u}(t)|: t_{0} \leq t \leq t_{0}+\tau\right\}$, which contradicts (9.2). In the same fashion, we cannot have $u\left(t_{0}+\tau_{2}\right)>0$; if it were the case, we could find an 
interval $\left[\tau_{1}, \tau_{3}\right]$ containing $\tau_{2}$ and $h_{1}>0$ such that for all $h \in\left(0, h_{1}\right], u_{h}\left(\left[\tau_{1}, \tau_{3}\right]\right)$ belongs to the interior of $K$. But, in this case, $\dot{u}_{h}$ converges uniformly to $\dot{u}$ in $C^{0}\left(\left[\tau_{1}, \tau_{3}\right]\right)$ and this contradicts again (9.2).

Thus, we assume that $\tau_{2}$ is strictly positive and that $u\left(t_{0}+\tau_{2}\right)$ vanishes.

We infer from (2.3) that

$$
\left|\eta^{m+1}\right| \leq \max \left(\left|\eta^{m}\right|,\left|\eta^{m-1}\right|\right)+2 C_{3} h .
$$

We now use (9.2). We can see that for all $m \leq m(h)$,

$$
\left|\eta^{m(h)}\right| \leq \max \left(\left|\eta^{m}\right|,\left|\eta^{m-1}\right|\right)+2 C_{3}(m(h)-m) h,
$$

so that

$$
\max \left(\left|\eta^{m}\right|,\left|\eta^{m-1}\right|\right) \geq\left|\eta^{m(h)}\right|-2 C_{3}(m(h)-m) h .
$$

If $\tau_{4}<\tau_{2}$ is such that

$$
\tau_{2}-\tau_{4} \leq \gamma / 4 C_{3}
$$

we can see that for all $m \in\left\{\left\lceil\tau_{4} / h\right\rceil, \ldots, m(h)\right\}$, the following estimate holds:

$$
\max \left(\left|\eta^{m}\right|,\left|\eta^{m-1}\right|\right) \geq \operatorname{ess} \sup \left\{|\dot{u}(t)|: t_{0} \leq t \leq t_{0}+\bar{\tau}\right\}+\gamma / 2 \text {. }
$$

But the function $\left|\dot{u}_{h}\right|$ converges almost everywhere on $\left[t_{0}, t_{0}+\tau\right]$ to $|\dot{u}|$, and so does $\max \left(\left|\dot{u}_{h}(\cdot-h)\right|,\left|\dot{u}_{h}\right|\right)$. Therefore, in the limit, relation (9.3) leads to

$$
\begin{gathered}
\liminf _{h \downarrow 0} \operatorname{ess} \sup \left\{\left|\dot{u}_{h}(t)\right|: t \in\left[t_{0}+\tau_{4}, t_{0}+\tau_{2}\right]\right\} \\
\geq \operatorname{ess} \sup \left\{|u(t)|: t_{0} \leq t \leq t_{0}+\bar{\tau}\right\}+\gamma / 2,
\end{gathered}
$$

which is a contradiction.

A corollary can be inferred imediately from this proposition and Proposition 3.4.

Corollary 9.2. For all admissible initial conditions $\left(t_{0}, u_{0}, p_{0}\right)$, there exists $\tau>0$ and a solution of (1.2), (1.3a), (1.3b), (1.4a), (1.4b), (1.5), (1.6), and (1.7) defined on $\left[t_{0}, t_{0}+\tau\right]$.

We have proved above the existence of a nonempty interval on which the numerical scheme converges to a solution of (1.2), (1.3a), (1.3b), (1.4a), (1.4b), (1.5), (1.6), and (1.7). On the other hand, Lemma 8.1 gives a priori estimates on the solution of such a problem.

We couple now the a priori estimates with the local convergence result to obtain a global result.

TheOREM 9.3. Let $R$ be strictly larger than $\left|v_{0}\right|$, and let $\tau(R)$ be given as in Lemma 8.1. Then, for all small enough $h$, the solution $y^{m}$ of the numerical scheme (1.10), (1.11), (1.12), (1.13) is defined on a discrete interval $\{0, \ldots, m(h)\}$ such that

$$
\liminf _{h \rightarrow 0} h m(h) \geq \tau(R)
$$

moreover, the approximation $u_{h}$ converges to a solution $u$ of the continuous time equation, i.e., (1.2), (1.3a), (1.3b), (1.4a), (1.4b), (1.5), (1.6), and (1.7), which is defined on $\left[t_{0}, t_{0}+\tau(R)\right]$. 
Proof. Let $A$ be given by

$$
A=\max \left(3 R+1, u_{0}+T(3 R+1)\right) .
$$

Let $\{0, \ldots, m(h)\}$ be the maximal discrete time interval for which the numerical scheme (1.10), (1.11), (1.12), (1.13) has a solution satisfying the estimate

$$
\forall m \in\{0, \ldots, m(h)-1\}, \quad\left|y^{m+1}-y^{m}\right| \leq A h .
$$

Let

$$
\tau_{1}=\liminf _{h \rightarrow 0} h m(h) .
$$

We know from Proposition 3.4 that $\tau_{1}$ is at least equal to some number $\tau>0$. Assume that $\tau_{1}$ is strictly inferior to $\tau(R)$. Proposition 9.1 implies in particular that for all $\varepsilon>0$

$$
\begin{aligned}
& \limsup _{h \rightarrow 0}\left\{\sup \left|\eta^{m}\right|: t_{0} \leq t^{m} \leq t_{0}+\tau_{1}-\varepsilon\right\} \\
& \quad \leq \operatorname{ess} \sup \left\{|\dot{u}(t)|: t_{0} \leq t \leq t_{0}+\tau_{1}-\varepsilon\right\} \leq R,
\end{aligned}
$$

thanks to the a priori estimates proved in Lemma 8.1. Since the above inequalities hold for all $\varepsilon>0$, we see that

$$
\limsup _{h \rightarrow 0}\left\{\sup \left|\eta^{m}\right|: t_{0} \leq t^{m} \leq t_{0}+\tau_{1}\right\} \leq R .
$$

Let

$$
a=\max \left(R+1 / 2, u_{0}+T(R+1 / 2)\right) ;
$$

Theorem 3.1 implies the existence of $\tau_{2}>0$ such that if $\widehat{y}^{0}$ and $\widehat{y}^{1}$ satisfy property $P(a, h)$, and $\widehat{t}_{0}$ is any time in $[0, T)$, then there exists a numerical solution of (1.12), (1.13) which satisfies

$$
\forall m \in\left\{0, \ldots,\left\lfloor\tau_{2} / h\right\rfloor\right\}, \quad\left|\widehat{y}^{m+1}-\widehat{y}^{m}\right| \leq A h .
$$

We denote

$$
l(h)=\left\lfloor\left(\tau_{1}-\tau_{2} / 2\right) / h\right\rfloor,
$$

and we initialize with the following choices:

$$
\widehat{t}_{0}=t_{0}+h l(h), \quad \widehat{y}^{0}=y^{l(h)}, \quad \widehat{y}^{1}=y^{l(h)+1} .
$$

With these data, we know that $\widehat{y}^{m}$ exists for $0 \leq m h \leq \tau_{2}$, so that the numerical solution $y^{m}$ is extended up to $\left\lfloor\left(\tau_{1}+\tau_{2} / 2\right) / h\right\rfloor-1$, and therefore,

$$
\liminf _{h \rightarrow 0} h m(h) \geq \tau_{1}+\tau_{2} / 2,
$$

which is a contradiction. 
10. Numerical experiments. In this section, we report about the numerical implementation of our scheme and of the impact detection method; we compare these results to results obtained for a penalized version of our model, using a freely available scientific computation package.

In view of its practical importance and of its ease of programming, we have limited ourselves to vibro-impact, i.e.,

$$
f(t, u, \dot{u})=a \cos \omega t-u-2 \alpha \dot{u}
$$

with constraint set

$$
K=\left[u_{\min },+\infty\right) .
$$

The following numerical values are kept constant throughout the numerical experiments:

$$
\alpha=0.5, \quad a=1, \quad \omega=50, \quad e=0.5 .
$$

Observe that $u_{\min } / a$ is the relevant parameter. We have observed in previous work [17], [19], [18] that if we systematically choose

$$
u(0)=u_{\min }, \quad \dot{u}(0)=0.1,
$$

the variation of the parameter $u_{\text {min }}$ triggers a variety of dynamical behaviors: periodic solutions, period doubling, and chaotic attractors.

All the penalty computations presented here have been implemented as SCILAB programs, a free high level scientific computation software developed and distributed by INRIA (http://www-rocq.inria.fr/scilab). Some of the other computations have been performed in FORTRAN.

10.1. Implementation particulars. The impact detection scheme goes as follows: starting from initial data $t_{j}, u\left(t_{j}\right)=u_{\min }, \dot{u}\left(t_{j}\right)$, the solution of the linear problem

$$
\ddot{u}+2 \alpha \dot{u}+u=a \cos \omega t
$$

is found explicitly; a nonlinear solver finds the first zero $t_{j+1}>t_{j}$ of $t \mapsto u(t)-u\left(t_{j}\right)$ and this instant is called $t_{j+1}$. We let

$$
u\left(t_{j+1}+0\right)=-e u\left(t_{j+1}-0\right),
$$

and we restart the process.

The foregoing description is slightly too rough: if there is an accumulation of impact instants, we have to define a threshold of velocity at $t_{j}$, under which we set the solution equal to $u_{\min }$, as long as $\cos \omega t$ remains negative. Observe that the detection method is potentially accurate to machine precision, since the nonlinear solvers for a scalar function are extremely precise, and the threshold velocity can be taken very small.

The numerical scheme is implemented as follows:

$$
\begin{aligned}
& y^{n+1}=-e y^{n-1}+\max \left((1+e) u_{\min }, x^{n}\right), \\
& x^{n}=\frac{h^{2} a}{1+\alpha h} \cos \left(\omega t_{n}\right)+\frac{2-h^{2}}{1+\alpha h} y^{n}-\frac{(1-e)-\alpha h(1+e)}{1+\alpha h} y^{n-1} .
\end{aligned}
$$




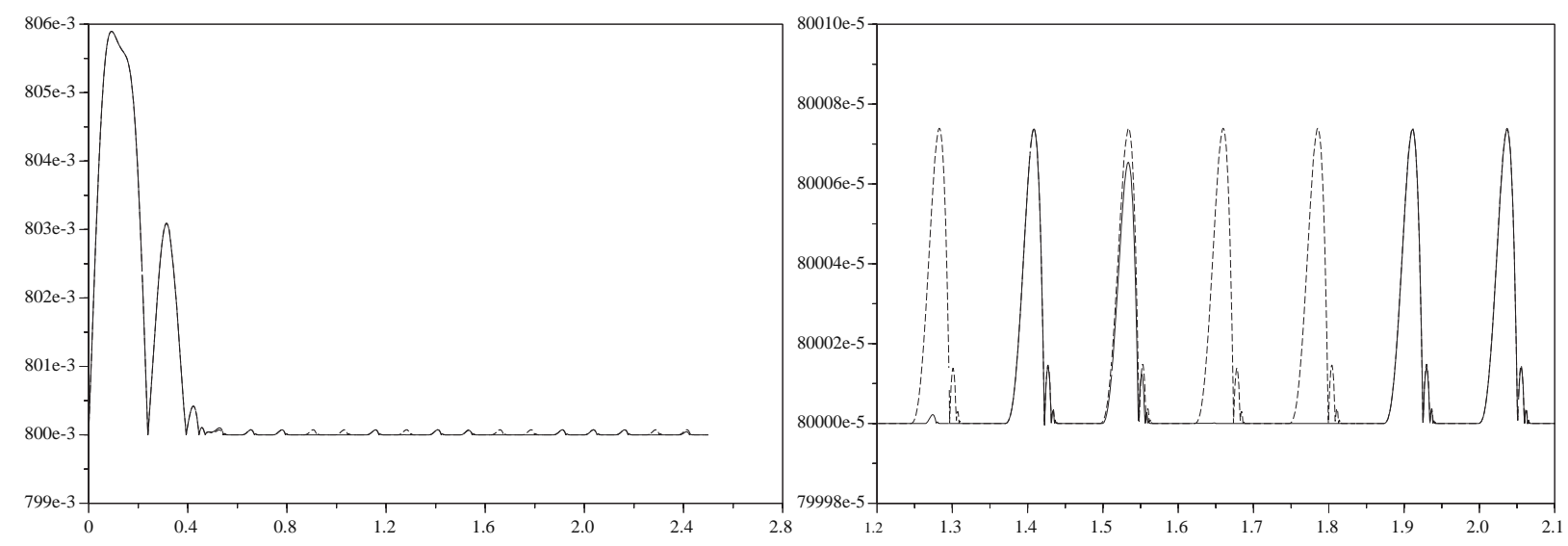

FIG. 10.1. The penalized solution on the left and its zoom on the right for time step $h=$ $1.2510^{-5}$ and $\varepsilon=10^{-6} / 256$.

The reader will check that this form is equivalent to (1.12) and (1.13); one of the advantages of this numerical scheme is that it is absolutely trivial to program.

For the penalty method, we applied the SCILAB function ode with the option stiff to the ordinary differential system

$$
\begin{aligned}
& \dot{u}=v \\
& \dot{v}=a \cos \omega t-u-2 \alpha v+\frac{\left(u-u_{\text {min }}\right)^{-}}{\varepsilon}+2 \frac{\beta \operatorname{sign}^{-}\left(u-u_{\text {min }}\right)}{\sqrt{\varepsilon}},
\end{aligned}
$$

where

$$
r^{-}=-\min (r, 0), \quad \operatorname{sign}^{-}(r)= \begin{cases}-1 & \text { if } r<0, \\ 0 & \text { otherwise }\end{cases}
$$

The parameter $\beta$ is defined in terms of the restitution coefficient $e$ by

$$
\beta=-\frac{\ln e}{\sqrt{\pi^{2}+(\ln e)^{2}}} .
$$

It has been proved in [21] that the solution of (10.1) converges to a solution of (1.2), (1.3a), (1.3b), (1.4a), (1.4b), and (1.5). The choice of $\beta$ is also justified in that reference.

10.2. Periodic solution: $\boldsymbol{u}_{\min }=\mathbf{0 . 8}$. The solution obtained by the scheme and impact detection agree satisfactorily: they both converge as time increases to a periodic solution, with an infinite number of impacts per period - of which we calculate only a finite number, of course!

The penalized approximation is satisfactory for not too small values of the penalty parameter, but for very small values of the parameter, it completely misses some of the periods (see Figure 10.1). The choice of parameters corresponds to $\sqrt{\varepsilon} / h=5$, which is quite sufficient for a good numerical approximation of the rebound.

As a matter of comparison we show in Figure 10.2 that the scheme and the detection method coincide very precisely.

We cannot offer much in the way of the explanations, since we did not go into the details of the SCILAB code to understand its inner workings; though it is an open package, with freely accessible code, we treated it as a black box. 


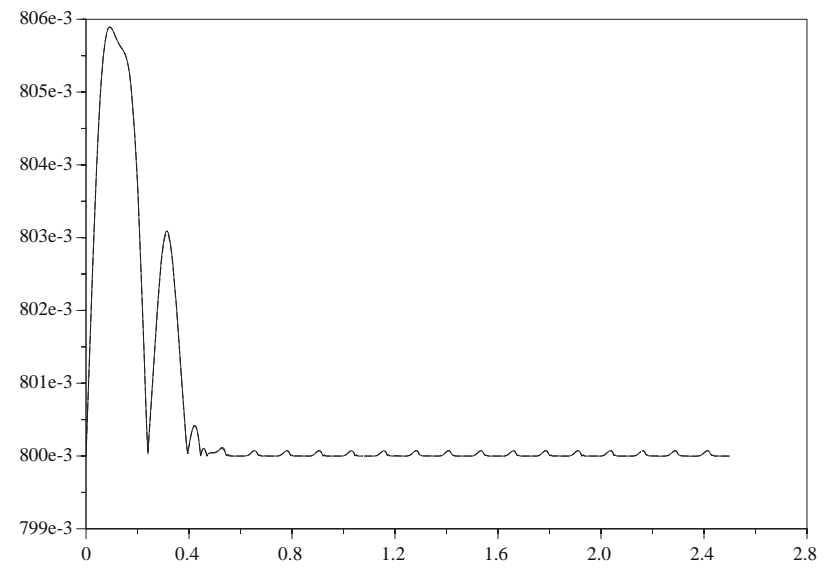

FIG. 10.2. The scheme for $h=0.0003125$ superposed to the solution by detection.
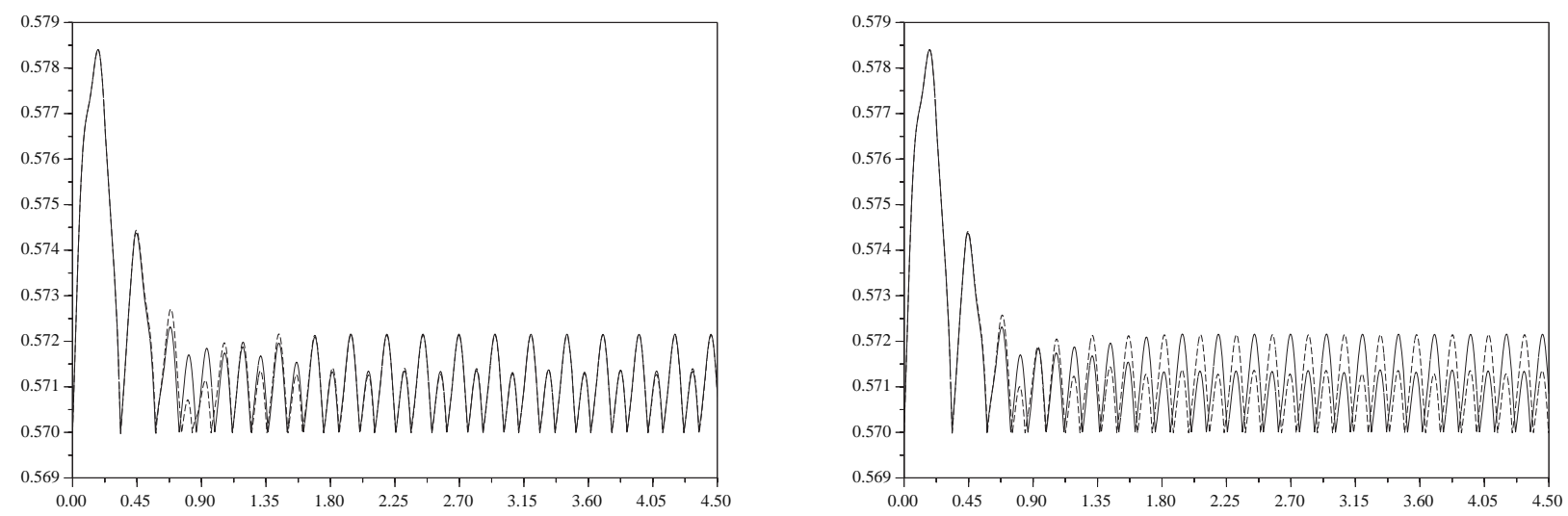

FIG. 10.3. On the left: detection (solid) and scheme (dotted); on the right: detection (solid) and penalty (dotted).

10.3. Period doubling: $\boldsymbol{u}_{\min }=\mathbf{0 . 5 7}$. We observe period doubling slightly above this value, and the scheme continues to agree satisfactorily with the detection method.

The penalty method gives also period doubling, but, depending on $\varepsilon$ and the time step, we may obtain either a good approximation of the period doubled solution obtained by the previous two approaches or its translate by a half (doubled) period, i.e., $2 \pi / \omega$.

For instance, Figure 10.3 shows the results of the calculation with the detection scheme, our ad hoc scheme with a time step $h=510^{-4}$, and the penalty method for $\varepsilon=10^{-7}$ with a time step $h=210^{-5}$.

The results of the numerical scheme do not seem to depend on the time step, once convergence is experimentally achieved; see Figure 10.4, left, where the solution is significantly improved by decreasing the time step from $6.2510^{-4}$ to $5.10^{-4}$. In contrast, on the right, decreasing the time step with the same penalty parameter $\varepsilon=10^{-6}$ does not give a significant improvement: for the larger time parameter, the beginning of the numerical solution is bad, and for the smaller one, we hit a phase shifted solution.

The penalized solution is also very sensitive to the choice of the penalty parameter; see Figure 10.5, with the same time step of $210^{-5}$ and penalty parameters of $10^{-7}$ and $10^{-8}$.

The results of the penalty method keep depending on the choice of the time step and $\varepsilon$ (see Figures 10.4, right, and 10.5), and we have not been able to establish the 

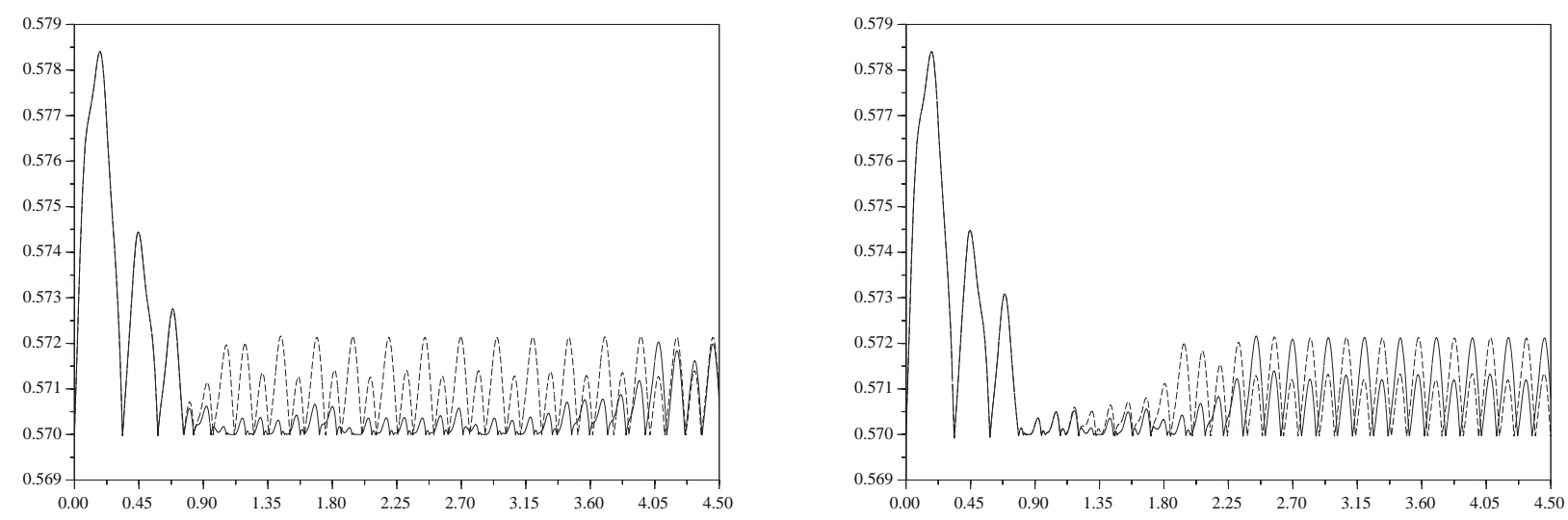

FIG. 10.4. Left: the scheme with $h=6.2510^{-4}$ (solid line) and with $h=510^{-4}$ (dashed line); right: the penalty method with $h=210^{-5}, \varepsilon=10^{-6}$ (solid line) and with $h=10^{-4}, \varepsilon=10^{-6}$ (dashed line).

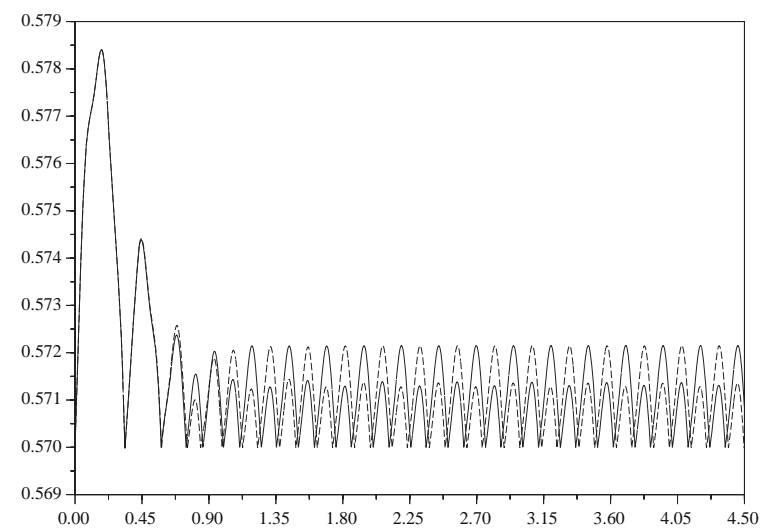

FIG. 10.5. The penalty method with $h=210^{-5}, \varepsilon=10^{-8}$ (solid line) and with $h=210^{-5}, \varepsilon=$ $10^{-7}$ (dashed line).

pattern of dependency.

10.4. Strange attractor: $\boldsymbol{u}_{\min }=\mathbf{0 . 5 4}$. We picture a stroboscopic view of the attractors by displaying the sequences

$$
(u((2 k+1) \pi / \omega), \dot{u}((2 k+1) \pi / \omega))
$$

for 1989 values of $k$.

Figure 10.6 displays a superposition of the computation by the detection method and the scheme, in FORTRAN double precision. Figure 10.7 displays a superposition of the SCILAB computation by the penalty method and by the scheme; it is somewhat surprising to see so few points of the scheme in this last computation, while the sizes of vectors are identical: $k$ varies from 1 to 1989, corresponding to a final time of 250 (and not to the bicentennial of the French Revolution). We believe that this phenomenon may be due to a bad control in SCILAB of the format of the numbers.

Nevertheless, these figures show a very satisfactory agreement between the three methods.

10.5. Numerical conclusion. We would like to stress the qualitative properties of numerical schemes in a dynamical systems framework: this scientific program has been started by several authors, and we refer to [31] for an overview of the subject. However, the methods of analysis rely heavily on a smoothness assumption which is not satisfied here, and therefore, they do not apply. Thus, at the present moment, we 


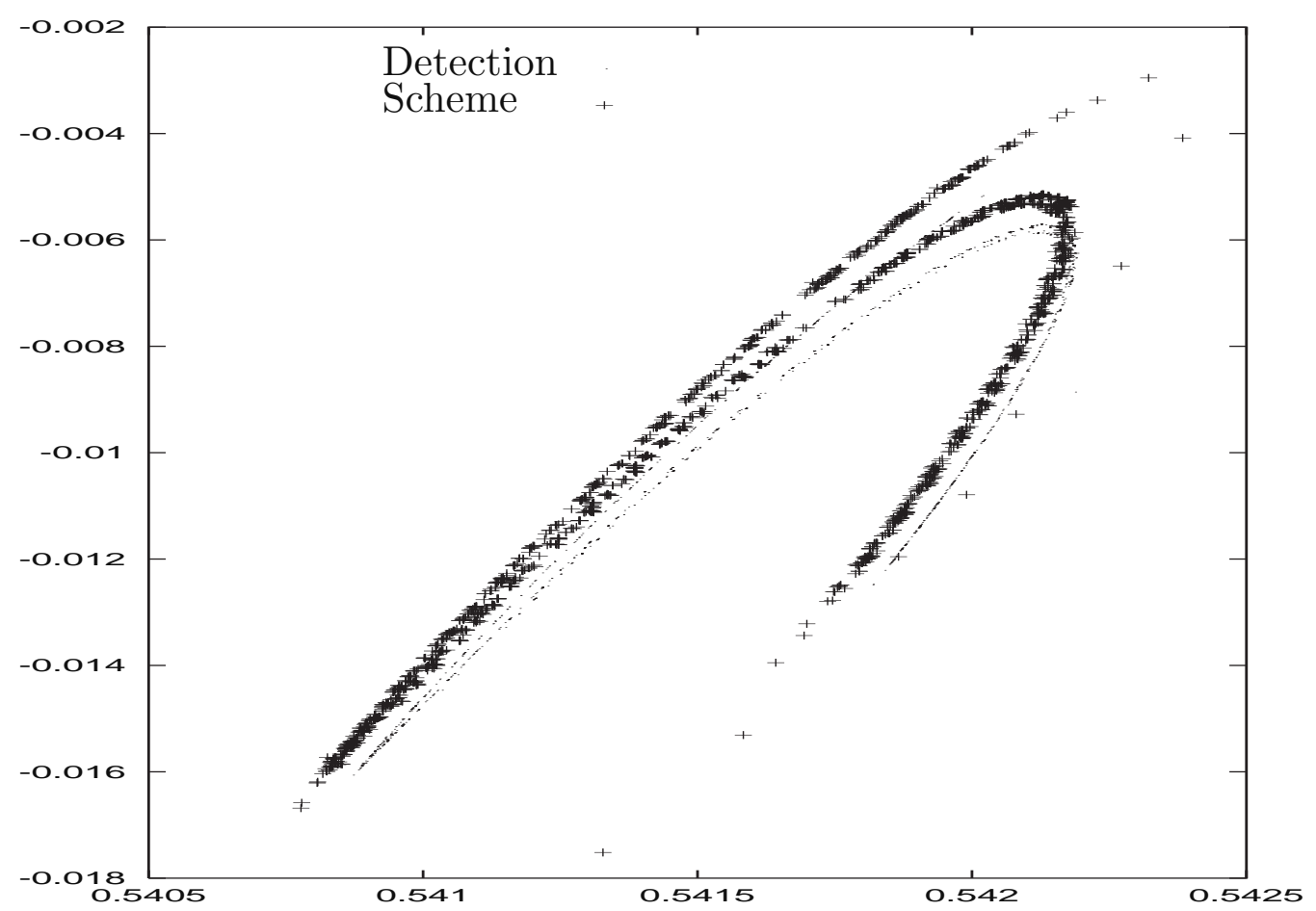

FIG. 10.6. A superposition of the stroboscopic pictures of the attractor obtained by the detection method (dots) and the scheme (points): FORTRAN calculation with time step $510^{-4}$.

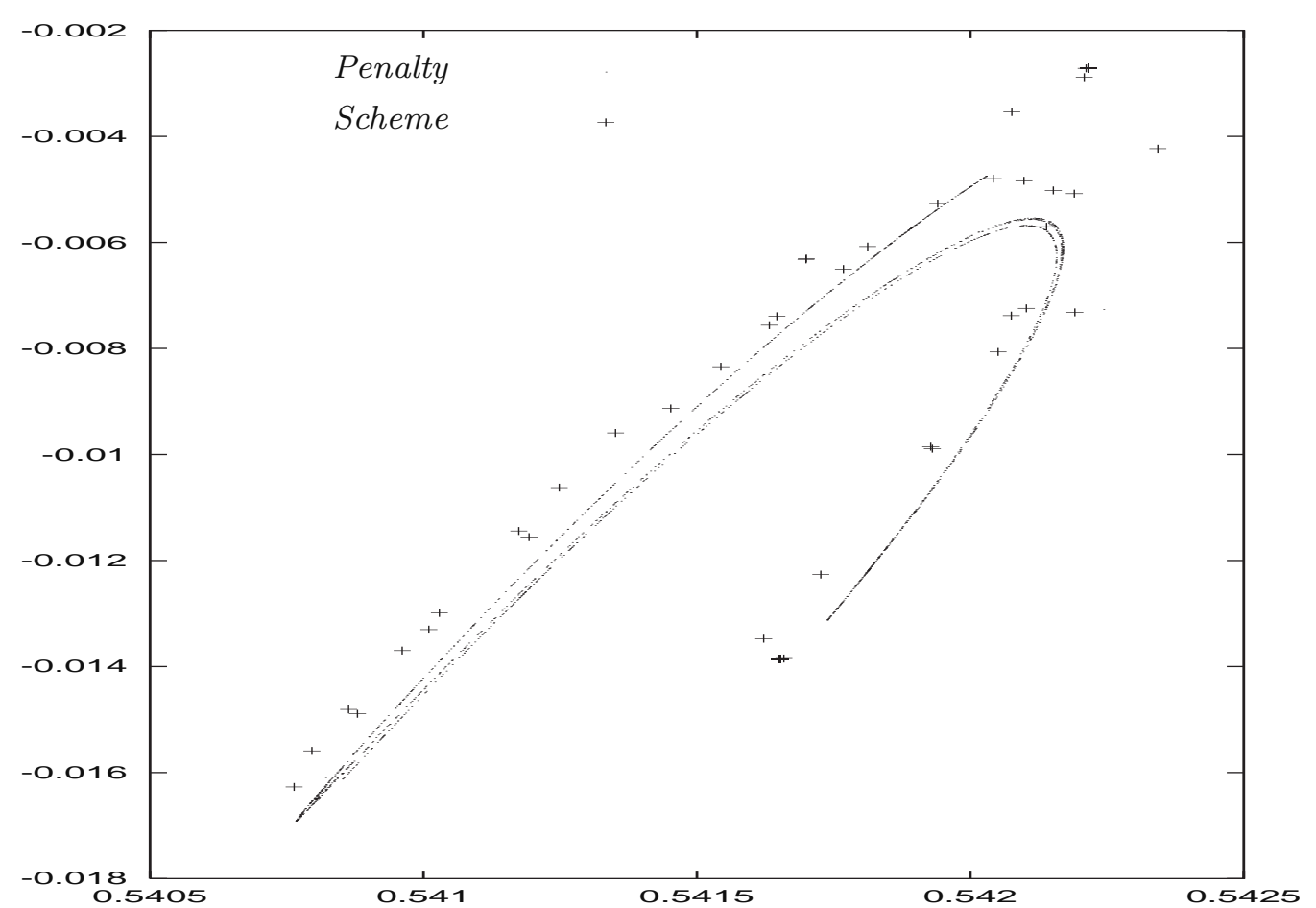

FIG. 10.7. A superposition of the stroboscopic pictures of the attractor obtained by the penalty method (dots) and the scheme (points): SCILAB calculation with time step $\pi / 2500$.

are reduced to experimental numerics, but we should keep in mind a rational approach to the numerical analysis of dynamical systems, focusing not only on the accuracy of a given method for finite time intervals but also on the qualitative properties of the method for long time ranges. The provisional conclusion for our one-dimensional case is that the penalty method performs reasonably well, but it misses details, while the 
detection method enables us to benchmark our ad hoc scheme and to certify that it does not miss details. Our scheme is quite easy to implement, and it contains no black box; however, it is still missing some bells and whistles, such as time step control. In any case, our scheme is of very low order: order one with respect to the position and order zero with respect to the velocity. The reason is that the velocity is discontinuous and the numerical impact times are usually distinct from their limit. Schemes with a better order of convergence should approximate very precisely the impact times, but one cannot but wonder whether it is really necessary to do so.

\section{REFERENCES}

[1] H. BrÉZIS, Analyse fonctionnelle. Théorie et applications, Masson, Paris, 1983.

[2] G. Buttazzo and D. Percivale, On the approximation of the elastic bounce problem on Riemannian manifolds, J. Differential Equations, 47 (1983), pp. 227-245.

[3] N. Dunford And J. T. Schwartz, Linear operators. Spectral theory. Selfadjoint operators in Hilbert space. Part II, John Wiley and Sons, New York, 1988.

[4] M. Kunze and M. D. P. Monteiro Marques, On the discretization of degenerate sweeping processes, Portugal. Math., 55 (1998), pp. 219-232.

[5] M. Laghdir and M. D. P. Monteiro Marques, Dynamics of a particle with damping, friction, and percussional effects, J. Math. Anal. Appl., 196 (1995), pp. 902-920.

[6] M. Laghdir and M. D. P. Monteiro Marques, Measure-differential inclusions in percussional dynamics, J. Convex Anal., 4 (1997), pp. 381-393.

[7] M. Mabrouk, Liaisons unilatérales et chocs élastiques quelconques: un résultat d'existence, C. R. Acad. Sci. Paris Sér. I Math., 326 (1998), pp. 1353-1357.

[8] M. Mabrouk, A unified variational model for the dynamics of perfect unilateral constraints, Eur. J. Mech. A Solids, 17 (1998), pp. 819-842.

[9] M. D. P. Monteiro Marques, Differential Inclusions in Nonsmooth Mechanical Problems. Shocks and Dry friction, Birkhäuser Verlag, Basel, 1993.

[10] M. D. P. Monteiro Marques, An existence, uniqueness and regularity study of the dynamics of systems with one-dimensional friction, Eur. J. Mech. A Solids, 13 (1994), pp. 277-306.

[11] J.-J. Moreau, Les liaisons unilatérales et le principe de Gauss, C. R. Acad. Sci. Paris, 256 (1963), pp. 871-874.

[12] J.-J. Moreau, Rafle par un convexe variable. I, Secrétariat des Math. 118, U.É.R. de Math., Univ. Sci. Tech. Languedoc, Montpellier, France, 1971.

[13] J.-J. Moreau, Rafle par un convexe variable. II, Secrétariat des Math. 122, Univ. Sci. Tech. Languedoc, Montpellier, France, 1972.

[14] J.-J. Moreau, Evolution problem associated with a moving convex set in a Hilbert space, J. Differential Equations, 26 (1977), pp. 347-374.

[15] J.-J. Moreau, Application of convex analysis to some problems of dry friction, in Trends in Applications of Pure Mathematics to Mechanics, Vol. II Pitman, Boston, 1979, pp. 263280.

[16] J.-J. Moreau, Liaisons unilatérales sans frottement et chocs inélastiques, C. R. Acad. Sci. Paris Sér. II Méc. Phys. Chim. Sci. Univers Sci. Terre, 296 (1983), pp. 1473-1476.

[17] M. Panet, L. Paoli, And M. Schatzman, Vibrations with an obstacle and a finite number of degrees of freedom, in Proceedings of the International Symposium on Identification of Nonlinear Mechanical Systems from Dynamic Tests-Euromech 280, L. Jezequel and C.-H. Lamarque, eds., Balkema, Rotterdam, 1992.

[18] M. Panet, L. Paoli, And M. Schatzman, Theoretical and numerical study for a model of vibrations with unilateral constraints, in Contact Mechanics, M. Raous, M. Jean, and J.-J. Moreau, eds., Plenum Press, New York, 1995, pp. 457-464.

[19] L. Paoli, Analyse numérique de vibrations avec contraintes unilatérales, Ph.D. thesis, Université Claude Bernard-Lyon 1, Lyon, France, 1993.

[20] L. Paoli and M. Schatzman, A numerical scheme for impact problems: II. The multidimensional case, SIAM J. Numer. Anal., 40 (2002), pp. 734-768.

[21] L. Paoli and M. Schatzman, Mouvement à un nombre fini de degrés de liberté avec contraintes unilatérales: cas avec perte d'énergie, M2AN Math. Model. Numer. Anal., 27 (1993), pp. 673-717.

[22] L. Paoli And M. Schatzman, Schéma numérique pour un modèle de vibrations avec contraintes unilatérales et perte d'énergie aux impacts, en dimension finie, C. R. Acad. Sc. Paris Sér 
I Math., 317 (1993), pp. 211-215.

[23] L. Paoli And M. Schatzman, Resonance in impact problems. Recent advances in contact mechanics, Math. Comput. Modelling, 28 (1998), pp. 385-406.

[24] D. Percivale, Bounce problem with weak hypotheses of regularity, Ann. Mat. Pura Appl. (4), 143 (1986), pp. 259-274.

[25] R. T. Rockafellar, Convex Analysis, Princeton University Press, Princeton, NJ, 1997.

[26] M. Schatzman, A class of nonlinear differential equations of second order in time, Nonlinear Anal., 2 (1978), pp. 355-373.

[27] D. E. Stewart, A numerical method for friction problems with multiple contacts, J. Austral. Math. Soc. Ser. B, 37 (1996), pp. 288-308.

[28] D. E. Stewart, Convergence of a time-stepping scheme for rigid-body dynamics and resolution of Painlevé's problem, Arch. Ration. Mech. Anal., 145 (1998), pp. 215-260.

[29] D. E. Stewart and J. C. Trinkle, An implicit time-stepping scheme for rigid body dynamics with inelastic collisions and Coulomb friction, Internat. J. Numer. Methods Engrg., 39 (1996), pp. 2673-2691.

[30] D. E. Stewart And J. C. Trinkle, Dynamics, friction, and complementarity problems, in Complementarity and Variational Problems (Baltimore, MD, 1995), SIAM, Philadelphia, PA, 1997, pp. 425-439.

[31] A. M. Stuart and A. R. Humphries, Dynamical Systems and Numerical Analysis, Cambridge University Press, Cambridge, UK, 1996. 